\title{
Laue Gamma-Ray Lenses for Space Astrophysics: Status and Prospects
}

\author{
Filippo Frontera ${ }^{1}$ and Peter von Ballmoos ${ }^{2}$ \\ ${ }^{1}$ Physics Department, University of Ferrara, Via Saragat 1, 44100 Ferrara, Italy \\ ${ }^{2}$ Centre d'Etude Spatiale des Rayonnements, 9, Avenue du Colonel Roche, 31028 Toulouse, France
}

Correspondence should be addressed to Filippo Frontera, frontera@fe.infn.it

Received 21 April 2010; Accepted 4 November 2010

Academic Editor: Stephen L. O’Dell

Copyright (C) 2010 F. Frontera and P. von Ballmoos. This is an open access article distributed under the Creative Commons Attribution License, which permits unrestricted use, distribution, and reproduction in any medium, provided the original work is properly cited.

We review feasibility studies, technological developments, and the astrophysical prospects for Laue lenses devoted to hard X/gamma-ray astronomy observations.

\section{Introduction}

Hard X-/soft gamma-ray astronomy is a crucial window for the study of the most energetic and violent events in the Universe. With the ESA INTEGRAL observatory [1] and the NASA Swift satellite [2], unprecedented sky surveys in the band beyond $20 \mathrm{keV}$ are being performed [3, 4]. As a consequence, hundreds of celestial sources have already been discovered, new classes of Galactic sources are being identified, and an overview of the extragalactic sky is available, while evidence of extended matter-antimatter annihilation emission from our Galactic center [5] and of Galactic nucleosynthesis processes have been also reported $[5,6]$. However, in order to take full advantage of the extraordinary potential of soft gamma-ray astronomy, a new generation of telescopes is needed. The current instrumentation has relied on the use of direct-viewing detectors with mechanical collimators (e.g., BeppoSAX/PDS, [7]) and, in some cases, with modulating aperture systems, such as coded masks (e.g., INTEGRAL/IBIS, [8]). These telescopes are penalized by their modest sensitivities, that improve at best as the square root of the detector surface. The only solution to the limitations of the current generation of gamma-ray instruments is the use of focusing optics. To study either the continuum emission or the nuclear line emission from celestial sources, Laue lenses, based on diffraction from crystals in a transmission configuration, are particularly suited to focus photons in the hard X-/soft gamma-ray
$(<1 \mathrm{MeV})$ domain. As we will show, they show imaging capabilities for on-axis sources.

With these lenses, we expect a big leap in both flux sensitivity and angular resolution. As far as the sensitivity is concerned, the expected increase is by a factor of at least 10-100 with respect to the best nonfocusing instruments of the current generation, with or without coded masks. Concerning the angular resolution, the increase is expected to be more than a factor 10 (from $\sim 15$ arcmin of the mask telescopes like INTEGRAL IBIS to less than 1 arcmin).

The astrophysical issues that are expected to be solved with the advent of these telescopes are many and of fundamental importance. A thorough discussion of the science case has been carried out in the context of the mission proposal Gamma Ray Imager (GRI), submitted to ESA in response to the first AO of the 'Cosmic Vision 2015-2025' plan [9] (but see also [10-12]). We summarize here some of these issues.

(i) Deep study of high-energy emission physics in the presence of super-strong magnetic fields (magnetars)

The XMM and INTEGRAL observed spectra of Soft Gamma Ray Repeaters [13], and Anomalous X-ray pulsars [14] leave unsolved the question of the physical origin of the high-energy component $(>100 \mathrm{keV})$. A better sensitivity at $E>100 \mathrm{keV}$ is needed. 
(ii) Deep study of high-energy emission physics in compact galactic objects and AGNs

A clue to the emission region and mechanism, along with the properties of the hidden black hole, can be obtained with the measurement of the high-energy cutoff and its relation with the power-law energy spectrum of the compact objects. The current observational status is far from clear (see, e.g., $[15,16])$. Much more sensitive observations are needed, for both AGNs and compact Galactic sources. In the case of blazars, the gamma-ray observations are crucial for the determination of their emission properties given that their energy emission peaks at hundreds of $\mathrm{keV}$ [17].

(iii) establishing the precise role of nonthermal mechanisms in extended objects like galaxy clusters

The existence of hard tails from Galaxy Clusters (GCs) is still a matter of discussion [18], and, should they exist, their origin is also an open issue. Are they the result of a diffuse emission or are they due to AGNs in the GC? In the former case, what is the emission mechanism? What is their contribution to $\mathrm{CXB}$ ? To answer these questions will require much more sensitive observations, like those achievable with broad band Laue lenses.

(iv) Origin of cosmic hard X/soft gamma-ray diffuse background

Currently, a combination of unobscured, Compton thin, and Compton thick radio-quiet AGN populations with different photon index distributions and fixed high-energy spectral cutoff $\left(E_{c}\right)$ are assumed in synthesis models of the Cosmic X-ray background (CXB) [19]. Is it reasonable to assume a fixed $E_{c}$ for these sources? A photon-energydependent contribution from radio-loud AGN to CXB, like blazars, is generally assumed. But their real contribution is still a matter of discussion. Deep spectral measurements of a significant sample of AGNs beyond $100 \mathrm{keV}$ is needed to solve these issues.

\section{(v) Positron Astrophysics}

Positron production occurs in a variety of cosmic explosion and acceleration sites, and the observation of the characteristic $511 \mathrm{keV}$ annihilation line provides a powerful tool to probe plasma composition, temperature, density, and ionization degree. The positron annihilation signature is readily observed from the galactic bulge region, yet the origin of the positrons remains mysterious. Compact objects-both galactic and extragalactic_-are believed to release significant numbers of positrons leading to $511 \mathrm{keV}$ gamma-ray line emission in the inevitable process of annihilation. A recent SPI/INTEGRAL all-sky map [5] of galactic $\mathrm{e}^{-} \mathrm{e}^{+}$annihilation radiation shows an asymmetric distribution of $511 \mathrm{keV}$ emission that has been interpreted as a signature of lowmass X-ray binaries with strong emission at photon energies $>20 \mathrm{keV}$ (hard LMXBs). A claim for an annihilation line from a compact source (Nova Muscae) was reported in the $90 \mathrm{~s}$ [20] but was never confirmed. Much more sensitive observations are needed to study the annihilation line origin, sources, and their nature.

\section{(vi) Physics of supernova explosions}

Type Ia supernovae (SNe Ia) are major contributors to the production of heavy elements and hence a critical component for the understanding of life cycles of matter in the Universe and the chemical evolution of galaxies. Because Laue lens telescopes allow the direct observation of radioactive isotopes that power the observable light curves and spectra, gamma-ray observations of SNe Ia that can be performed with this type of instrument are in a position to allow a breakthrough on the detailed physical understanding of SNe Ia. This is important for its own sake, but it is also necessary to constrain systematic errors when using high-z $\mathrm{SNe}$ Ia to determine cosmological parameters.

High-resolution gamma-ray spectroscopy provides a key route to answering these questions by studying the conditions in which the thermonuclear explosion starts and propagates. A sensitivity of $10^{-6}$ photons $\mathrm{cm}^{-2} \mathrm{~s}^{-1}$ to broadened gamma-ray lines allows observations of supernovae out to distances of $50-100 \mathrm{Mpc}$. Within this distance, it is expected that there will always be a type Ia $\mathrm{SN}$ in the phase of gamma-ray line emission, starting shortly after explosion, and lasting several months.

In this paper, we review the physical principles of Laue lenses, their geometry, their optimization criteria, their optical properties, the current development status and the prospects for future missions for gamma-ray astronomy.

\section{Laue Lens Concept}

Diffraction lenses use the interference between the periodic nature of the electromagnetic radiation and a periodic structure such as the matter in a crystal. For a classical textbook on X-ray diffraction see, for example, [21]. In a Laue lens, the photons pass through the full crystal, using its entire volume for interacting coherently. In order to be diffracted, an incoming gamma-ray must satisfy the Braggcondition, relating the spacing of lattice planes $d_{h k l}$ with the energy of incident photons $E$ and with the angle of incidence $\theta_{B}$ with respect to the chosen set of planes $(h k l)$ (The indices $h, k, l, k n o w n$ as Miller indices, are defined as the reciprocals of the fractional intercepts which the lattice plane makes with the crystallographic axes. For example, if the Miller indices of a plane are ( $h k l)$, written in parentheses, then the plane makes fractional intercepts of $1 / h, 1 / k, 1 / l$ with the axes, and, if the axial lengths of the unit cell are $a, b, c$, the plane makes actual intercepts of $a / h, b / k, c / l$. If a plane is parallel to a given axis, its fractional intercept on that axis is taken as infinity and the corresponding Miller index is zero. If the Miller indices [ hkl] are shown in square brackets, they give the direction of the plane with the same indices.)

$$
2 d_{h k l} \sin \theta_{B}=n \frac{h c}{E}
$$

where $d_{h k l}$ (in $\AA$ ) is the spacing of the lattice planes $(h k l), n$ is the diffraction order, $h c=12.4 \mathrm{keV}$. $\AA$ and $E$ is the energy (in $\mathrm{keV}$ ) of the gamma-ray photon. An elementary illustration of the Bragg condition, in two different configurations (reflection and transmission), is given in Figure 1, where 


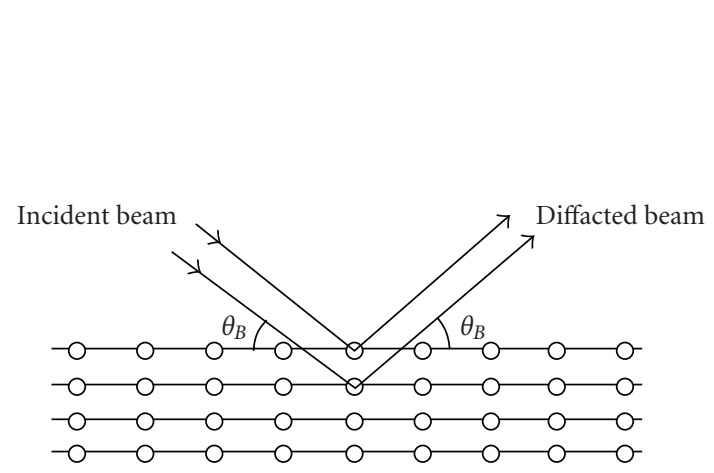

(a)

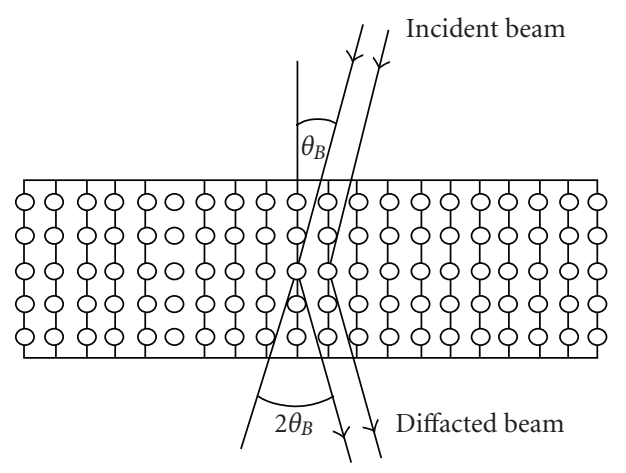

(b)

FIGURE 1: The Bragg condition for constructive interference of a gamma-ray photon beam with the atoms of a given crystalline plane. (a) Bragg diffraction in reflection configuration (Bragg geometry). (b) Bragg diffraction in transmission configuration (Laue geometry).

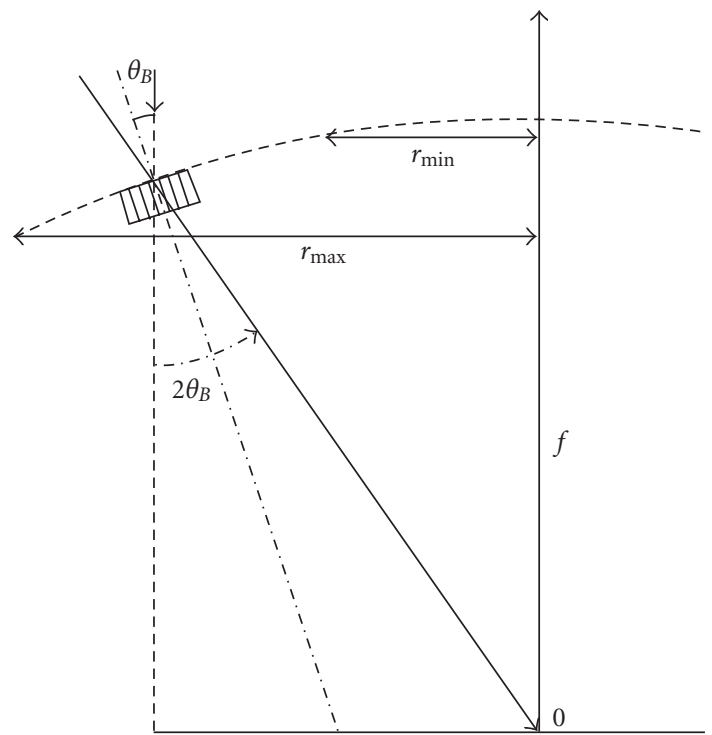

FIgURE 2: Geometry of a Laue lens (see text).

it can be seen that the incident waves are reflected by the parallel planes of the atoms in the crystal.

A Laue lens is made of a large number of crystals, in transmission configuration (Laue geometry), that are disposed such that they will concentrate the incident radiation onto a common focal spot. A convenient way to visualize the geometry of a crystal lens is to consider it as a spherical cup covered with crystal tiles having their diffracting planes perpendicular to the sphere (see Figure 2). The focal spot is on the symmetry axis at a distance $f=R / 2$ from the cup, with $R$ being the radius of the sphere of which the spherical cup is a part; $f$ is called the focal length.

From the Bragg equation, for the first diffraction order $(n=1)$, it can be seen that the photons incident on a given crystal at distance $r\left(r_{\min } \leq r \leq r_{\max }\right)$ from the lens axis can be reflected toward the lens focus if their energy $E$ is given by

$$
E=\frac{h c}{2 d_{h k l}} \sin \left[\frac{1}{2} \arctan \left(\frac{f}{r}\right)\right] \approx \frac{h c f}{d_{h k l} r},
$$

where the approximated expression is valid for gamma-ray lenses, given the small diffration angles involved.

Conversely, the lens radius $r$ (see Figure 2) at which the photon energy $E$ is reflected in the focus is given by

$$
r=f \tan \left[2 \theta_{B}\right] \approx \frac{h c f}{d_{h k l} E} .
$$

Rotation around the lens optical axis at constant $r$ results in concentric rings of crystals (see Figure 3(a)), while a uniformly changing value of $r$ gives rise to an Archimedes spiral (Figure 3(b)). Assuming that the chosen diffracting planes $(h k l)$ of all the lens crystals are the same, in the first case (constant $r$ ), the energy of the diffracted photon will be centered on $E$ for all the crystals in the ring, while in the second case (Archimedes spiral), the reflected energy $E$ will continuously vary from one crystal to the other, as shown in Figure 4.

2.1. Energy Passband. Any Laue lens will diffract photons over a certain energy passband $\left(E_{\min }, E_{\max }\right)$. From (2), at firstorder diffraction (the most efficient), it results that

$$
\begin{aligned}
& E_{\min } \approx \frac{h c f}{d_{h k l} r_{\max }}, \\
& E_{\max } \approx \frac{h c f}{d_{h k l} r_{\min }} .
\end{aligned}
$$

Given that, for astronomical applications, the lens passband is desired to be covered with the highest effective area (The effective area at energy $E$ is defined as the geometrical area of the lens projected in the focal plane times, the total reflection efficiency at energy E.) and in a smooth manner as a function of energy, the energy bands of the photons reflected by contiguous crystal rings or, in the case of the Archimedes structure of a lens, by contiguous crystals have to overlap each other, like in Figure 4. Since the full width at half maximum (fwhm) of the acceptance angle $\delta$ (known as the Darwin width) of perfect crystals is extremely narrow (fractions of an arcsec to a few arcsec, see [21]), such materials are not suitable for astrophysical Laue lenses. In order to increase 


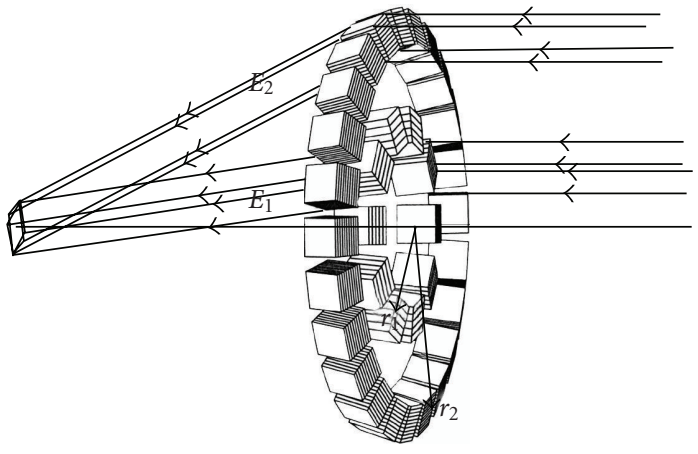

(a)

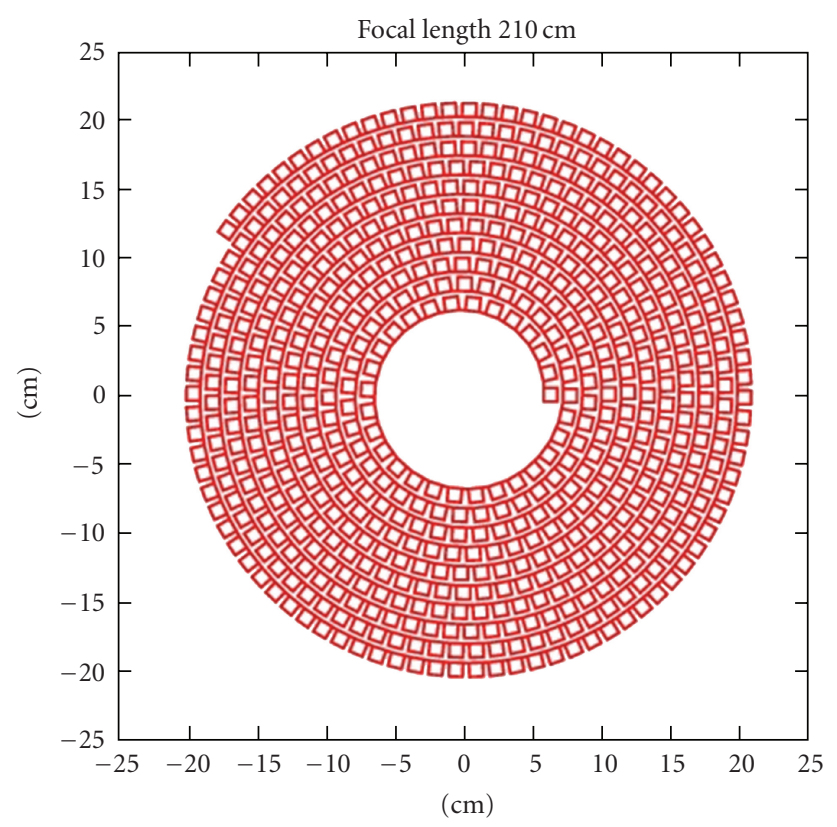

(b)

Figure 3: The basic design of a crystal diffraction lens in Laue geometry. Flat crystal tiles are assumed. (a) concentric rings of a given radius $r$ concentrating a constant energy E. (b) crystal tiles disposed along an Archimedes' spiral result in a continuously varying energy E. Given the footprint of the crystals, the image in the focal plane has a minimum size equal to that of the crystal size.

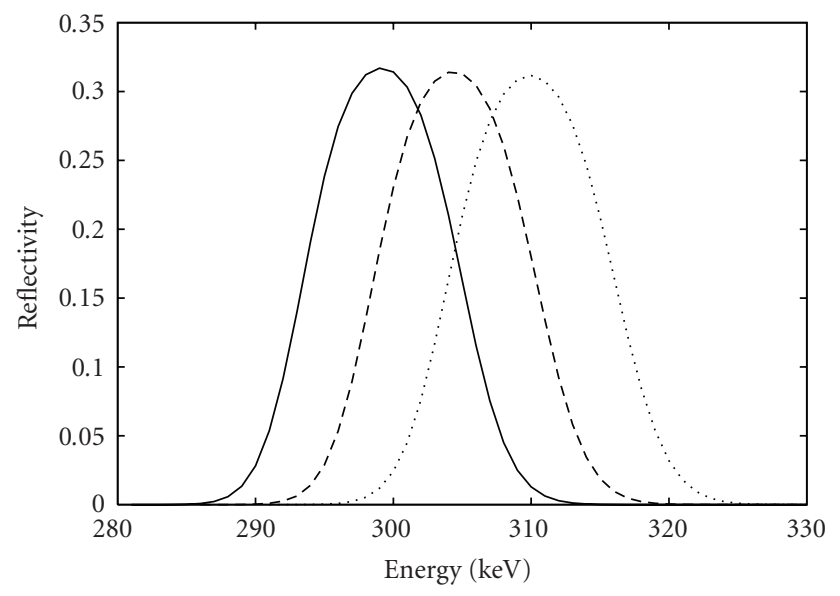

FIGURE 4: An example of the expected reflectivity profile of three contiguous crystals with a mosaicity of $1.5 \mathrm{arcmin}$ along an Archimedes' spiral. Reprinted from [22].

the energy passband of individual crystals, one uses mosaic crystals or curved crystals (see Section 3). The mosaicity of mosaic crystals (see Section 3.1) and the total bending angle of curved crystals (see Section 3.2) govern the flux throughput, the angular resolution, and the energy passband of the Laue lenses. The diffracted flux from a continuum source increases with increasing the mosaicity of mosaic crystals or the total bending angle of curved crystals. For a crystal lens telescope, crystals with mosaicities or total bending angles ranging from a few tens of arcseconds to a few arcminutes are of interest.
The bandwidth of a lens for an on-axis source is determined by the mosaicity or total bending angle of the individual crystals and by the accuracy of the alignment of the single crystals. By forming the derivative of the Bragg relation in the small angle approximation $\left(2 d_{h k l} \theta_{B} \approx n h c / E\right)$, we get

$$
\frac{\Delta \theta_{B}}{\theta_{B}}=\frac{\Delta E}{E} .
$$

If $\Delta \theta_{B}$ is the mosaicity of the mosaic crystal or the total bending angle of the curved crystal, the corresponding energy passband $\Delta E$ of the crystal becomes

$$
\Delta E=\frac{2 d_{h k l} \cdot E^{2} \cdot \Delta \theta_{B}}{n h c} .
$$

It is worth pointing out that, whereas the energy passband of a crystal lens grows with the square of energy, the Doppler broadening of the astrophysical lines (e.g., in SN ejecta) increases linearly with energy for a given expansion velocity.

\section{Crystal Reflectivity}

Both mosaic crystals and curved crystals are suitable to be used for a Laue lens. We discuss the properties of both of them and their reflectivity.

3.1. Mosaic Crystals. Mosaic crystals are made of many microscopic perfect crystals (crystallites) with their lattice planes slightly misaligned with each other around a mean direction, corresponding to the mean lattice planes $(h k l)$ 
chosen for diffraction. In the lens configuration assumed, the mean lattice plane is normal to the surface of the crystals. The distribution function of the crystallite misalignments from the mean direction can be approximated by a Gaussian function

$$
W(\Delta)=\frac{1}{\sqrt{2 \pi} \eta} \exp \left(-\frac{\Delta^{2}}{2 \eta^{2}}\right),
$$

where $\Delta$ is the magnitude of the angular deviation from the mean, while $\beta_{m}=2.35 \eta$ is the fwhm of the mosaic spread (called mosaicity).

For the Laue geometry and diffracting planes perpendicular to the cross section of the crystal tile (see, e.g., Figure 2), the crystal reflectivity $R(\Delta, E)$ is given by [21]

$$
\begin{aligned}
R(\Delta, E) & =\frac{I_{d}(\Delta, E)}{I_{0}} \\
& =\sinh (\sigma T) \exp \left[-\left(\mu+\gamma_{0} \sigma\right) \frac{T}{\gamma_{0}}\right] \\
& =\frac{1}{2}\left(1-e^{-2 \sigma T}\right) e^{-\mu T / \gamma_{0}},
\end{aligned}
$$

where $I_{0}$ is the intensity of the incident beam, $\mu$ is the absorption coefficient corresponding to that energy, $\gamma_{0}$ is the cosine of the angle between the direction of the photons and the normal to the crystal surface, $T$ is the thickness of the mosaic crystal, and $\sigma$ is

$$
\sigma=\sigma(E, \Delta)=W(\Delta) Q(E) f(A),
$$

where

$$
Q(E)=r_{e}^{2}\left|\frac{F_{h k l}}{V}\right|^{2} \lambda^{3} \frac{1+\cos ^{2}\left(2 \theta_{B}\right)}{2 \sin 2 \theta_{B}},
$$

in which $r_{e}$ is the classical electron radius, $F_{h k l}$ is the structure factor, inclusive of the temperature effect (Debye-Waller's factor), $V$ is the volume of the crystal unit cell, $\lambda$ is the radiation wavelength, and $\theta_{B}$ is the Bragg angle for that particular energy, while $f(A)$ in (9) is well approximated by

$$
f(A)=\frac{B_{0}(2 A)+\left|\cos 2 \theta_{B}\right| B_{0}\left(2 A\left|\cos 2 \theta_{B}\right|\right)}{2 A\left(1+\cos ^{2} \theta_{B}\right)} .
$$

Here, $B_{0}$ is the Bessel function of zero order integrated between 0 and $2 A$, with $A$ defined as follows:

$$
A=\frac{\pi t_{0}}{\Lambda_{0} \cos \theta_{B}}
$$

in which $t_{0}$ is the crystallite thickness, and $\Lambda_{0}$ (extinction length) is defined for the symmetrical Laue case (see e.g., $[23])$ as

$$
\Lambda_{0}=\frac{\pi V \cos \theta_{B}}{r_{e} \lambda\left|F_{h k l}\right|\left(1+\left|\cos 2 \theta_{B}\right|\right)} .
$$

In general, $f(A)<1$ and converges to 1 if $t_{0} \ll \Lambda_{0}$. In this case, we get the highest reflectivity.

The quantity $\gamma_{0} \sigma$ is known as secondary extinction coefficient and $T / \gamma_{0}$ is the distance travelled by the direct beam inside the crystal.
3.2. Curved Crystals. Similarly to mosaic crystals, curved crystals have an angular dispersion of the lattice planes and thus a much larger energy passband (see (6)) than perfect crystals. The properties of these crystals and the methods to get them are discussed in [24]. Here, we summarize their reflection properties.

The most recent theory of the radiation diffraction in transmission geometry for such crystals, in the case of a large and homogeneous curvature, is now well fixed and has been compared with the experimental results (see $[25,26])$. In this theory, the distortion of diffracting planes is described by the strain gradient $\beta_{s}$, that, in the case of a uniform curvature, is given by

$$
\beta_{s}=\frac{\Omega}{T_{0}(\delta / 2)},
$$

where $\Omega$ is the total bending angle and corresponds to the mosaicity of the mosaic crystals, $T_{0}$ is the thickness of the crystal and $\delta$ is the Darwin width.

When the strain gradient $\left|\beta_{s}\right|$ becomes larger than a critical value $\beta_{c}=\pi /\left(2 \Lambda_{0}\right)$, it has been shown that, for a uniform curvature of planes, the peak reflectivity $R^{\max }$ of a curved crystal is given by

$$
\begin{aligned}
R^{\text {peak }}\left(c_{p}, E\right) & =\frac{I_{r}^{\text {peak }}\left(c_{p}, E\right)}{I_{0}} \\
& =\left(1-e^{-\left(\pi^{2} d_{h k l}\right) /\left(c_{p} \Lambda_{0}^{2}\right)}\right) e^{-(\mu \Omega) /\left(c_{p} \cos \theta_{B}\right)},
\end{aligned}
$$

where $I_{r}^{\text {peak }}$ is the reflected peak intensity, $c_{p}=\Omega / T_{0}$ is the curvature of the lattice planes assumed to be uniform across the crystal thickness, and the extinction length $\Lambda_{0}=\Lambda_{0}(E)$ is given by (13). The reflected intensity profile $I_{r}\left(c_{p}, E\right)$ is that of a perfect crystal with the Darwin width replaced with $\Omega$. This profile is shown in Figure 5.

From the last equation, it can be shown that the highest peak reflectivity is obtained for a curvature of the lattice planes given by

$$
c_{p}^{\mathrm{opt}}=\frac{M}{\ln (1+M / N)},
$$

where $M=\pi^{2} d_{h k l} / \Lambda_{0}^{2}$ and $N=\mu \Omega / \cos \theta_{B}$.

3.3. Mosaic Crystals versus Curved Crystals. Both mosaic crystals and curved crystals can be used for a Laue lens, if they can be produced with the needed angular spread. However, in principle, curved crystals can reach a higher efficiency than mosaic crystals. Indeed, while the diffraction efficiency of mosaic crystals is limited to $50 \%$, that of curved crystals can reach $100 \%$. Another advantage of curved crystals is that the diffraction profile of a curved crystal is rectangular with width related to $\Omega$, while that of mosaic crystals is Gaussian with fwhm equal to the mosaicity $\beta_{m}$. Given the absence of Gaussian tails, curved crystals concentrate the flux better (see Section 4). This better performance of the curved crystals with respect to the mosaic crystals for Laue lenses is discussed in depth in [27]. 


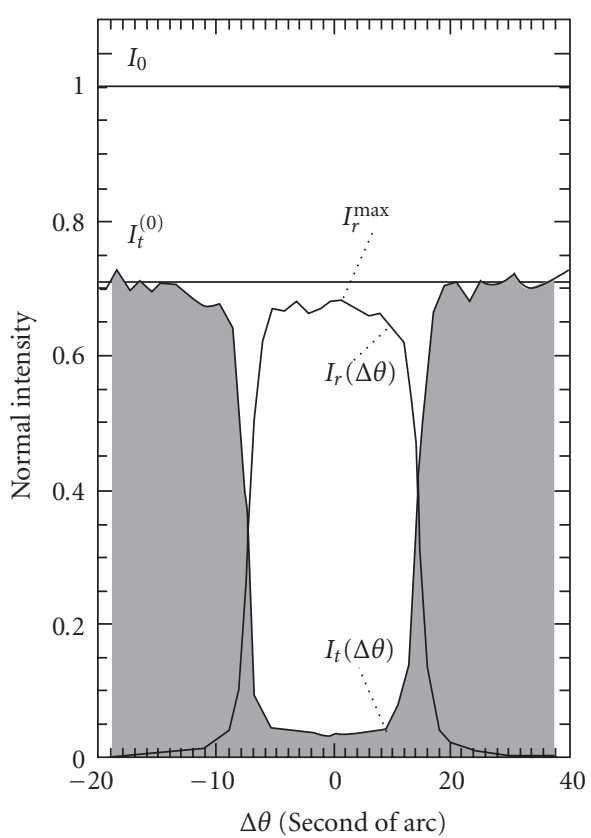

FIGURE 5: Reflectivity profile of a curved crystal as a function of the rocking angle $\Delta \theta$. This angle gives the difference between the incidence angle of the monochromatic photon beam and the Bragg angle. $I_{0}$ represents the incident intensity, $I_{t}(\Delta \theta)$ the transmitted intensity, and $I_{r}(\Delta \theta)$ the reflected intensity. Reprinted from [25].

Curved crystals can be obtained in various ways [24]. The most feasible techniques to be used for Laue lenses include the elastic bending of a perfect crystal (the technique commonly adopted in synchrotron radiation facilities), the deposition of a coating on a wafer, the growing of a twocomponent crystal whose composition varies along the crystal growth axis (see, e.g., [25]), or the indentation of one face of a wafer. The last technique is being developed at the University of Ferrara (V. Guidi, private communication) with very satisfactory results (see Figure 6). Also the deposition of a coating on a wafer is being tested at the same university.

\section{Optimization of a Laue Lens}

The free parameters of a Laue lens are the crystal properties (materials, lattice planes for diffraction, microcrystal size and mosaicity in the case of mosaic crystals, total bending angle in the case of curved crystals, and crystal thickness), the lens geometry (ring-like or Archimedes' spiral), its focal length and its nominal energy passband. Many optimization studies of these parameters have been performed and tested [24, 2931 .

4.1. Crystal Material. Independently of the crystal structure (mosaic or curved), in order to optimize the crystal reflectivity it is important to maximize $Q(E)$ as defined in (10). This is the integrated crystal reflectivity per unit volume, whose normalization is the ratio $\left|F_{h k l} / V\right|^{2}$ between the structure factor of the chosen lattice planes $F_{h k l}$ and the volume $V$ of the unit cell. The inverse of $V$ is the atomic density $N$. Thus,

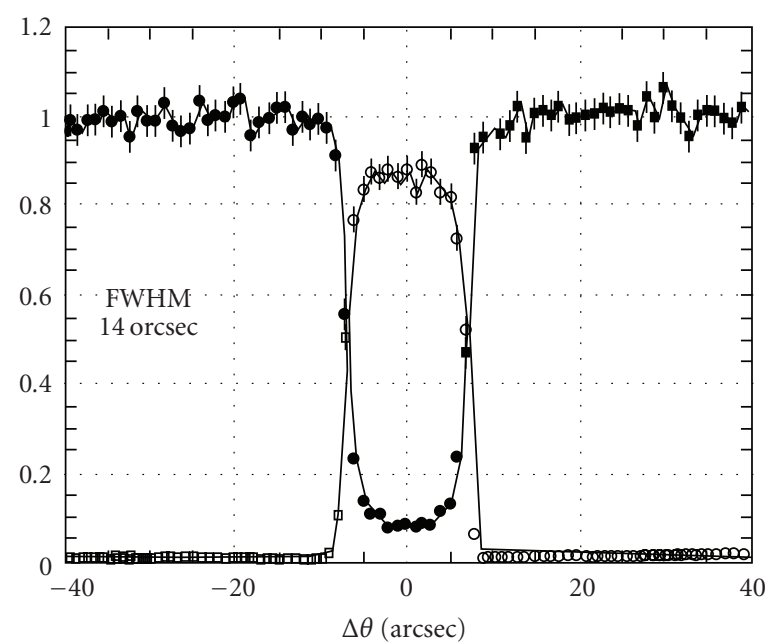

FIGURE 6: Measured rocking curve, in transmission geometry at $150 \mathrm{keV}$, of a $\mathrm{Si}(111)$ crystal curved at the University of Ferrara (see text). $\Delta \theta$ gives the difference between the incidence angle of the monochromatic photon beam and the Bragg angle. Open circles: ratio between measured intensity of the diffracted beam and measured intensity of the transmitted beam (also called diffraction efficiency). Filled circles: difference between transmitted and diffracted intensities. Note that the angle $\Delta \theta$, through the Bragg law, is related to the reflected photon energy. Thus, the figure also shows the energy bandwidth of the curved crystal. Reprinted from [28], who tested the crystal sample.

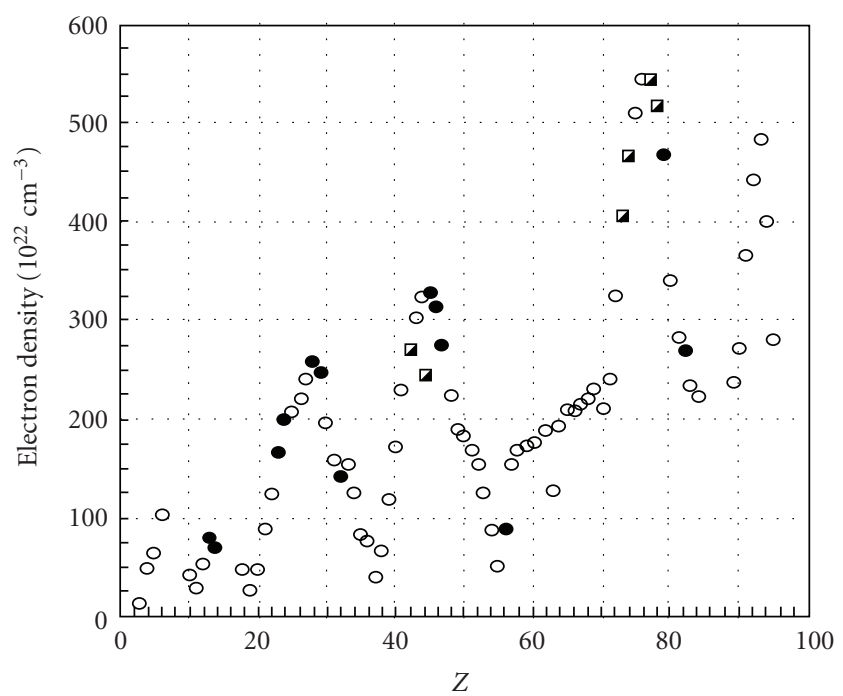

Figure 7: Density of a crystal unit cell versus element atomic number. Reprinted from [24].

a small $V$ (or a high $N$ ) is important for maximizing $Q(E)$. The value of $N$ as a function of the element atomic number $Z$ is shown in Figure 7.

As can be seen, for single-element materials, broad density peaks are apparent in correspondence of the atomic numbers $5,13,28,45$, and 78 . Common materials like $\mathrm{Al}$ $(Z=13), \mathrm{Si}(Z=14), \mathrm{Ni}(Z=28), \mathrm{Cu}(Z=29), \mathrm{Zn}$ 


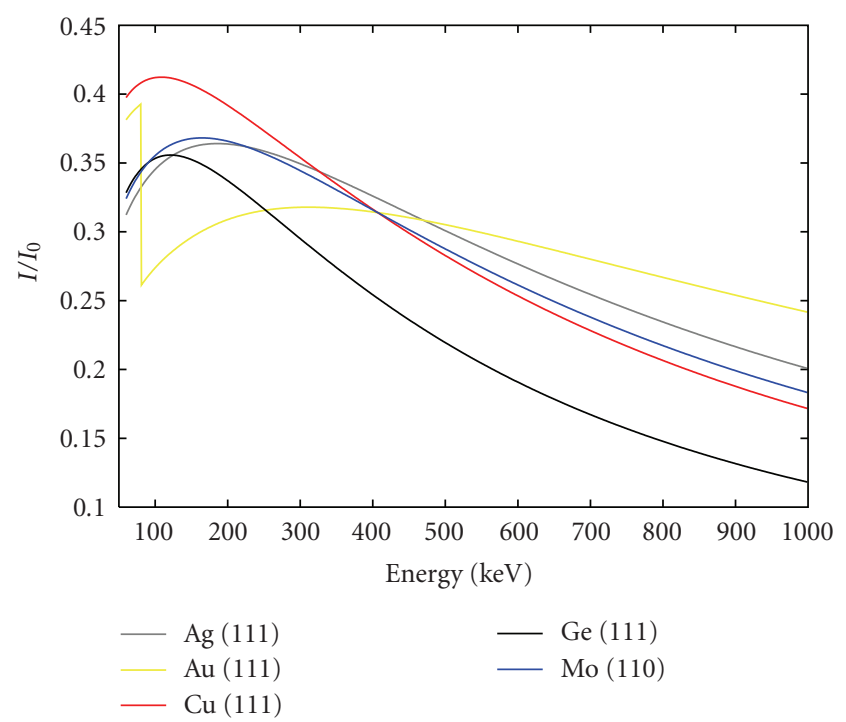

Figure 8: Peak reflectivity of 5 candidate crystal materials. The Miller indices used give the highest reflectivity. A mosaicity of $40 \mathrm{arcsec}$ is assumed. The thickness has been optimized. The production technology of mosaic crystals with the required spread is already mature for $\mathrm{Ge}$ and $\mathrm{Cu}$.

$(Z=30)$, Ge $(Z=32)$, Mo $(Z=42)$, Rh $(Z=45)$, $\mathrm{Ag}(Z=47), \mathrm{Ta}(Z=73), \mathrm{W}(Z=74)$, and $\mathrm{Au}(Z=$ $42)$ are good candidates to be used for Laue lenses and should be preferred to other elements if they are available as crystals with the requested properties. The peak reflectivity versus energy of few single-element mosaic crystal materials is shown in Figure 8.

Also double-element crystal materials can be used for Laue lenses. Several of them, developed for other applications, are already available, like GaAs, InAs, $\mathrm{CdTe}$, and $\mathrm{CaF}_{2}$. With some improvements, these crystal materials can be used for Laue lenses (see discussion in [24]).

Clearly the best lattice planes are those that optimize the structure factor $\left|F_{h k l}\right|$, under the condition that the corresponding $d_{h k l}$ is consistent with lens constraints, such as energy passband, lens size, and focal length (see below).

\subsection{Crystallite Size and Angular Distribution in Mosaic} Crystals. From the reflectivity (8), the crystallite thickness $t_{0}$ plays an important role in the reflectivity optimization. For fixed values of the mosaicity and crystal thickness, the highest reflectivity is obtained for a crystallite thickness that satisfies the condition $t_{0} \ll \Lambda_{0}$. In general, this implies a thickness of the order of $1 \mu \mathrm{m}$.

Unfortunately, this condition is still not always satisfied. From extended tests performed on $\mathrm{Cu}(111)$ supplied by ILL [32], it was found that the condition above is satisfied in single points [22], but not when the entire crystal cross section is irradiated (values even higher than $100 \mu \mathrm{m}$ have been found [24]). In addition, in [24], it is found that $t_{0}$ is energy dependent, which is a surprising result that requires an interpretation (see discussion therein).
The crystal mosaicity is another crucial parameter for the optimization of the lens performance. It can be seen [11, 2931 that, even if a higher mosaicity gives a larger lens effective area (see Figure 9), a higher spread also produces a larger defocusing of the reflected photons in the focal plane and thus a lower lens sensitivity.

This can be seen by introducing the focusing factor $G(E)$ of a Laue lens

$$
G(E)=f_{\mathrm{ph}} \frac{A_{\mathrm{eff}}(E)}{A_{d}}
$$

in which $A_{\text {eff }}(E)$ is the effective area of the lens and $A_{d}$ is the area of the focal spot which contains a fraction $f_{\mathrm{ph}}$ of photons reflected by the lens.

Assuming that the detector noise is Poissonian, it can be easily shown that $G(E)$ is inversely proportional to the minimum detectable continuum intensity of a lens

$$
I_{\min }(E)=\frac{n_{\sigma}}{\eta_{d} G} \sqrt{\frac{2 B}{A_{d} \Delta T \Delta E}},
$$

where $I_{\min }$ is given in units of photons $\mathrm{cm}^{-2} \mathrm{~s}^{-1} \mathrm{keV}^{-1}$ in the interval $\Delta E$ around $E, n_{\sigma}$ is the significance level of the signal (typically $n_{\sigma}=3-5$ ), $B$ is the focal plane detector background intensity (counts $\mathrm{cm}^{-2} \mathrm{~s}^{-1} \mathrm{keV}^{-1}$ ), $\Delta T$ is exposure time to a celestial source, and $\eta_{d}$ is the focal plane detector efficiency at energy $E$. For a lens made of mosaic crystals of $\mathrm{Cu}(111)$, Figure 10 shows the dependence of $G$ on focal length in two different energy bands, for different values of the mosaicity.

As can be seen, in spite of the fact that a higher spread gives a higher effective area, a lower spread gives a higher $G$ and thus a higher lens sensitivity. This effect is small at short focal lengths (10-20 m), but it becomes very significant at long focal lengths $(>30 \mathrm{~m})$, especially at high energies $(\sim 500 \mathrm{keV})$.

4.3. Crystal Thickness. The crystal thickness is another crucial parameter for the reflectivity optimization of a mosaic crystal. The best crystal thickness is given by

$$
T_{\text {best }}=\frac{1}{2 \sigma} \ln \left(1+\frac{2 \sigma \gamma_{0}}{\mu}\right),
$$

in the case of a mosaic crystal, and it is given by

$$
T_{\text {best }}=\frac{\Omega \ln (1+M / N)}{M},
$$

in the case of a curved crystal, where the involved quantities are defined in the sections above. In the case of a mosaic crystal, the optimum crystal thickness for various materials is shown in Figure 11.

As can be seen, the best crystal thickness depends on the absorption coefficient $\mu$. A high-absorption coefficient implies a low crystal thickness for the reflectivity optimization. 


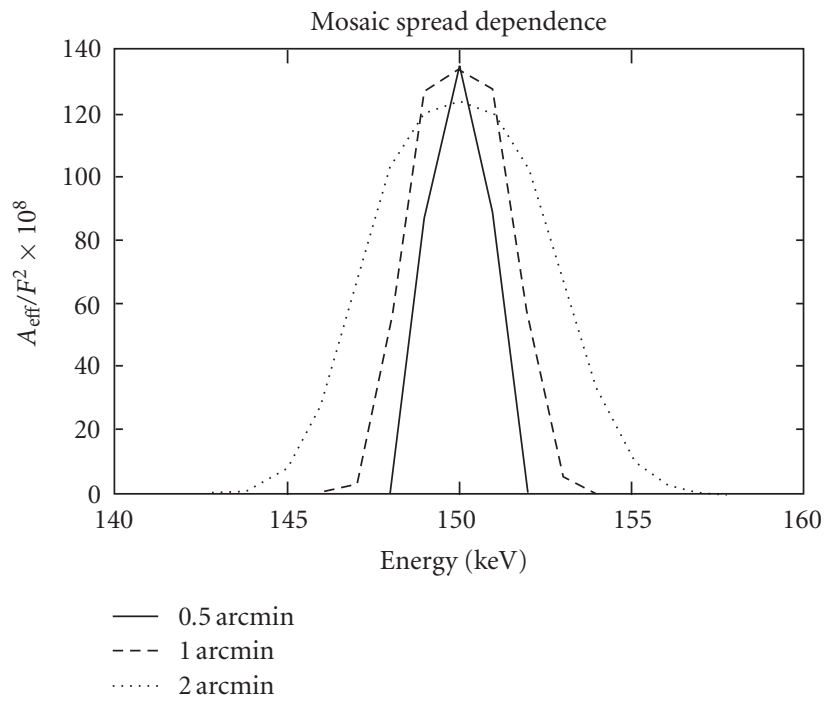

(a)

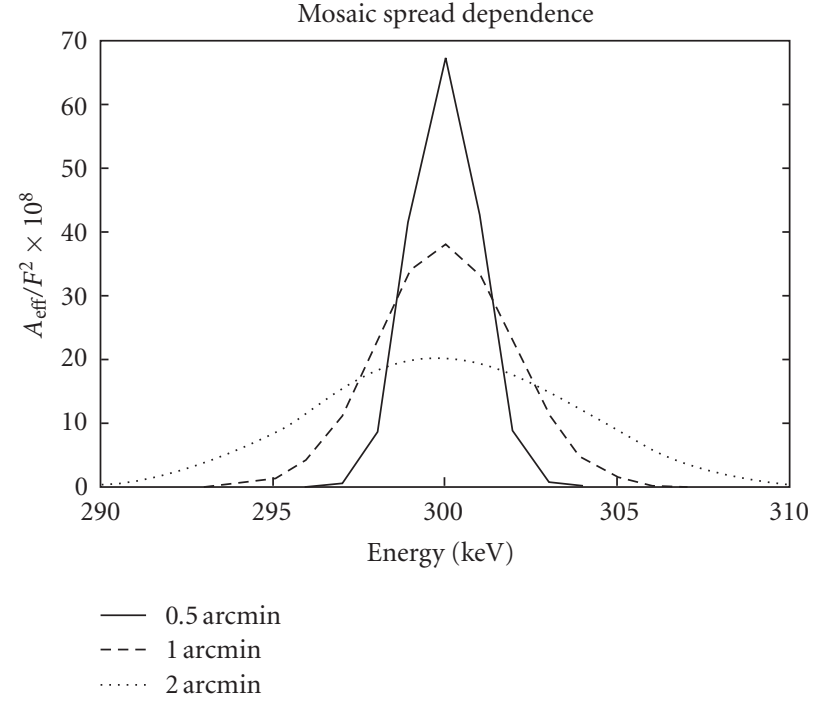

(b)

Figure 9: Normalized effective area for different values of the mosaicity of $\mathrm{Cu}(111)$. (a) First diffraction order; (b) second diffraction order. Reprinted from [31].

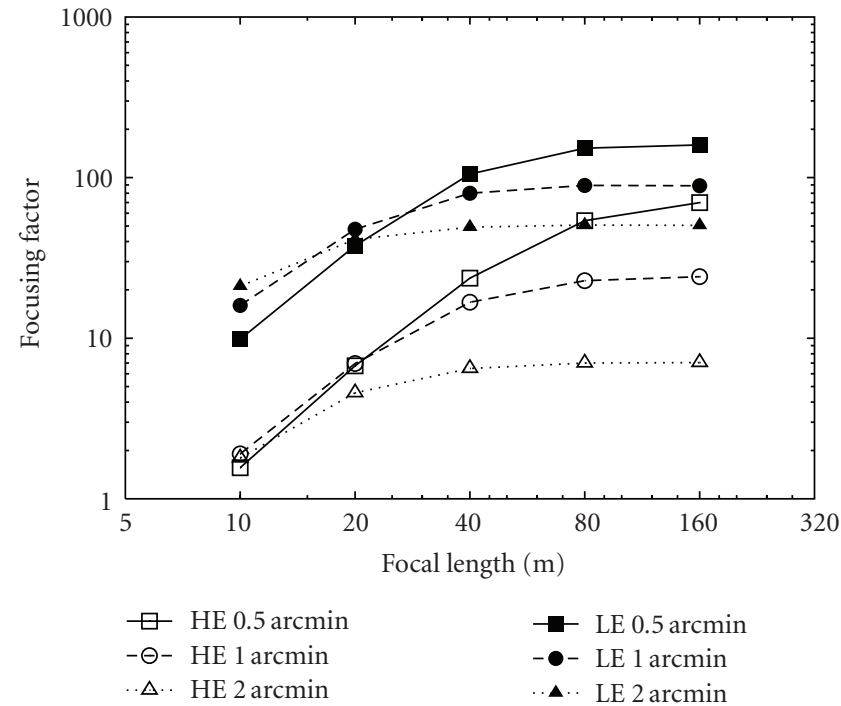

Figure 10: Dependence of the focusing factor $G$ of a lens made of mosaic crystals of $\mathrm{Cu}(111)$ on the focal length $f$ for three different values of the mosaicity in two different energy bands: $90-110 \mathrm{keV}$ (LE) and 450-550 keV (HE). Reprinted from [11].

4.4. Focal Length. The focal length has a key importance in the case of Laue lenses, more than its importance in the case of traditional focusing telescopes. Indeed, given that the energy passband of a single crystal is quite narrow (see e.g., Figure 4), from the expression of nominal energy passband $\left(E_{\min }, E_{\max }\right)$ of a lens (see $\left.(4)\right)$, it results that, for first-order diffraction, the lens radii $\left(r_{\min }, r_{\max }\right)$, needed to get a given passband, depend linearly on $f$

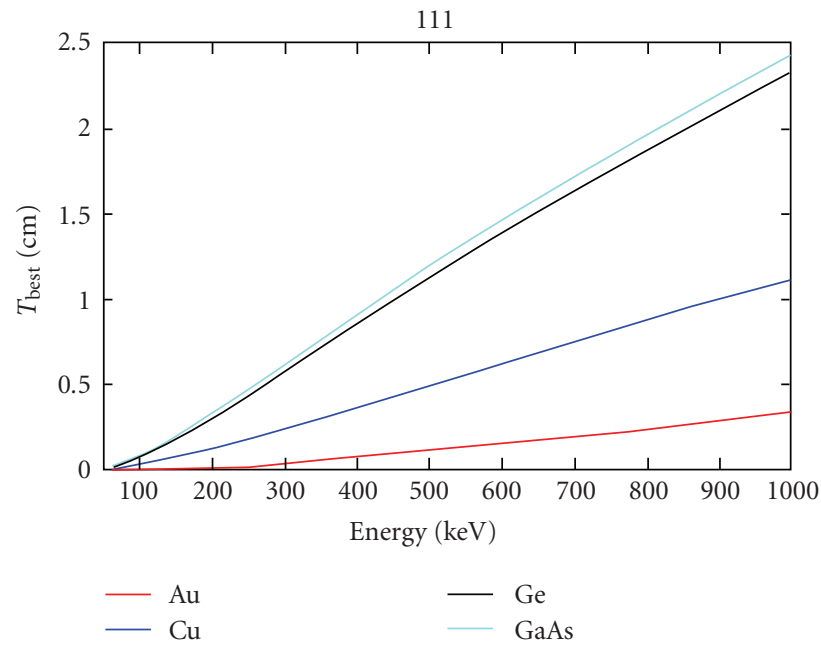

FIgURE 11: The best crystal thickness that maximizes the crystal reflectivity, for various materials. The mosaicity assumed is 1 arcmin, the crystallite thickness is $1 \mu \mathrm{m}$, and the crystal plane chosen for all of them is (111).

$$
\begin{aligned}
& r_{\min } \approx \frac{h c f}{d_{h k l} E_{\max }}, \\
& r_{\max } \approx \frac{h c f}{d_{h k l} E_{\min }} .
\end{aligned}
$$

Given that high energy photons are focused by the innermost part of the lens, to get a given lens inner area, the focal length must be increased (the lens area approximately increases with $\left.f^{2}\right)$.

A gamma-ray lens with a broad continuum coverage from $300 \mathrm{keV}$ to $1.5 \mathrm{MeV}$ was proposed in the 90s by Lund 


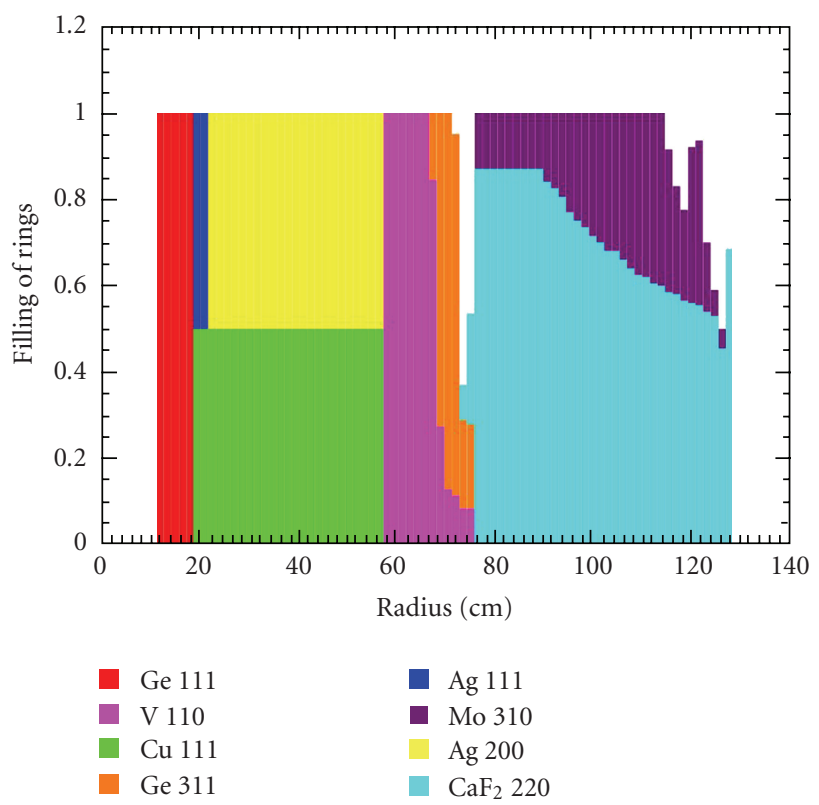

(a)

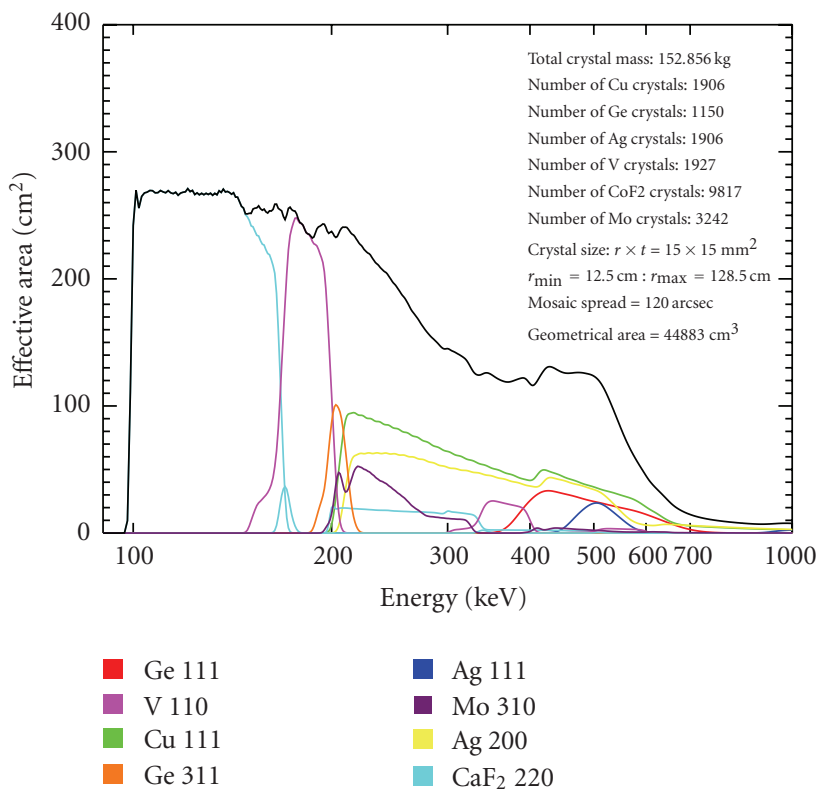

(b)

FIGURE 12: (a) Distribution of various flat mosaic crystals and chosen lattice planes in an example of a lens and focal length, as studied by Barriére et al. [28]. (b) Effective area of the studied lens. Colors show the contribution of each material.

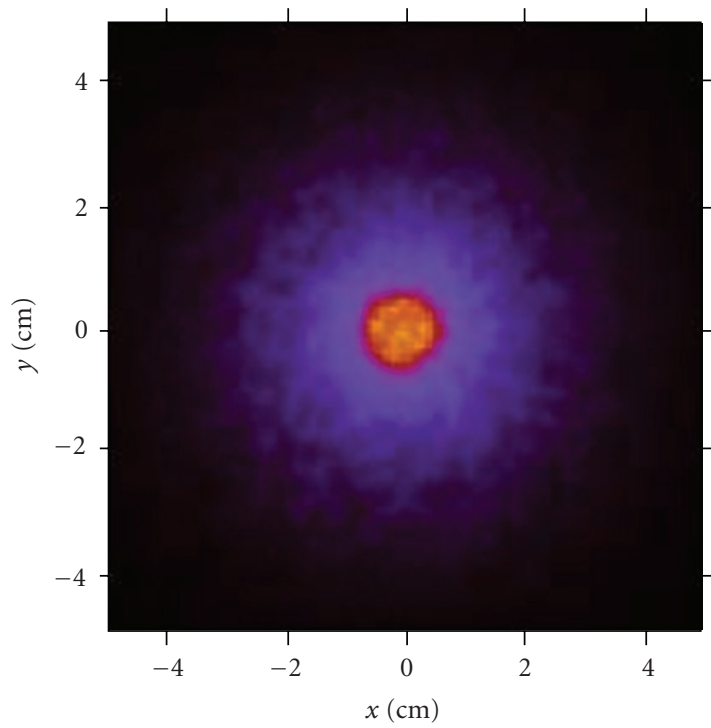

(a)

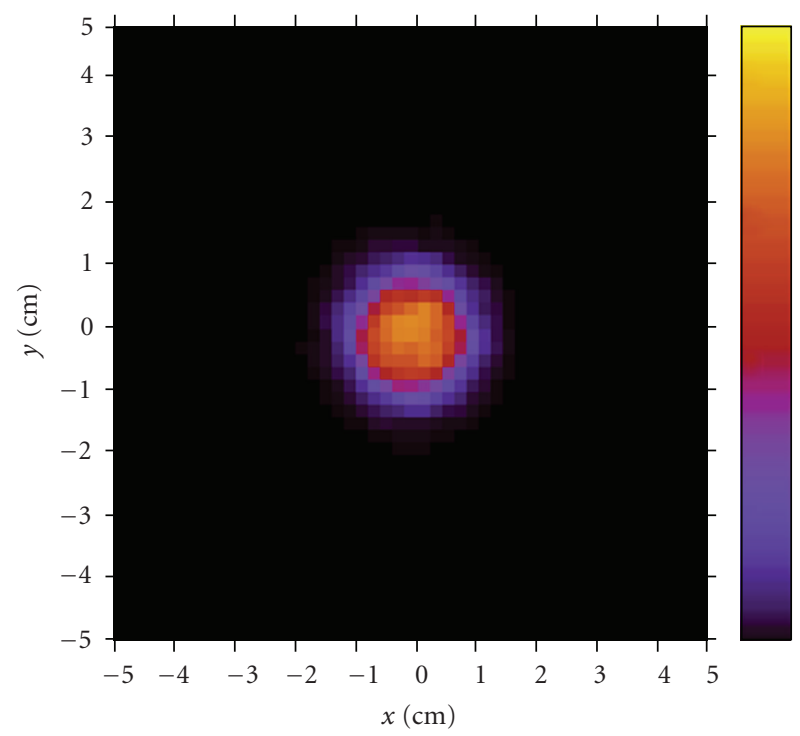

(b)

FIGURE 13: On-axis response function (PSF) of lenses made of flat mosaic crystals. (a) Lens with $40 \mathrm{~m}$ focal length for an on-axis source in the $150-600 \mathrm{keV}$ energy band. (b) Lens with $6 \mathrm{~m}$ focal length for an on-axis source in the 70-300 keV energy band. See text. Reprinted from $[33,34]$.

[35]. He assumed mosaic crystals of Copper and Gold. In order to achieve a significant effective area at high energies $\left(350 \mathrm{~cm}^{2}\right.$ at $300 \mathrm{keV}$ and $25 \mathrm{~cm}^{2}$ at $\left.1.3 \mathrm{MeV}\right)$, the focal length proposed was $60 \mathrm{~m}$.

4.5. Broad versus Narrow Passband Laue Lenses. For the lens optimization, the selection criteria of the crystal material and lattice planes can change depending on the requested lens passband. Two classes of Laue lenses can be identified, broad passband and narrow passband. The former covers a broad energy band (e.g., 100-600 keV) for the study of continuum source spectra, while the latter achieves an optimal sensitivity in a relatively narrow energy band (e.g., $800-900 \mathrm{keV}$ ) for gamma-ray line spectroscopy. These two classes of lenses 


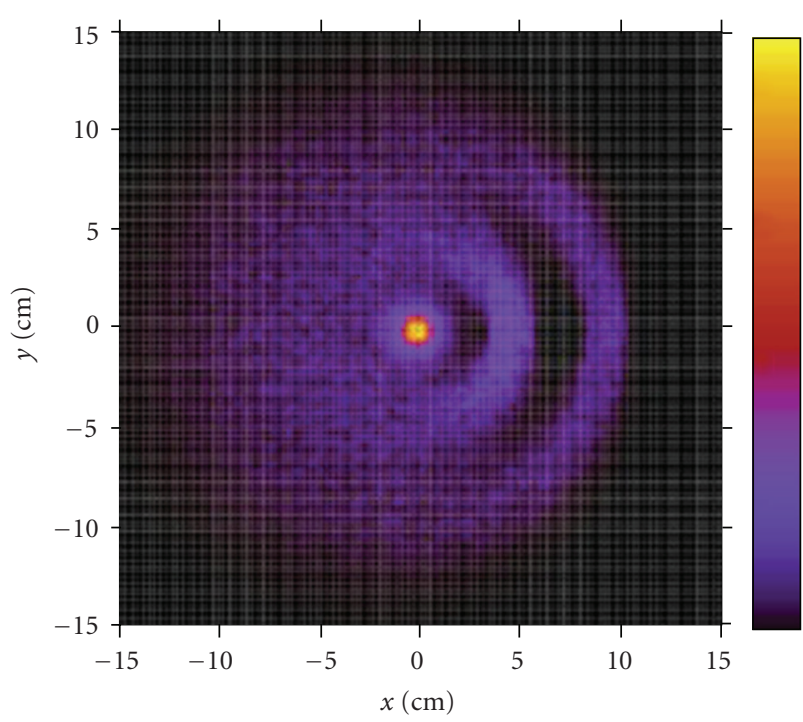

Figure 14: Off-axis expected response function (PSF) of a Laue lens made of flat mosaic crystals with $40 \mathrm{~m}$ focal length in the 150$600 \mathrm{keV}$ energy band. Three-point sources are simulated, at 0,2 and 4 arcmin off-axis. See text. Reprinted from [34].

require different criteria in the crystal choice and disposition in the lens for its optimization.

4.5.1. Narrow Passband Laue Lenses. Assuming a ring-like geometry, these lenses use a different crystalline plane $(h k l)$ for every ring in order to diffract photons in only one energy band centered on the energy $E_{0}$. If, for a given focal length, the ring at distance $r_{0}$ from the lens axis concentrates photons centered at energy $E_{0}$ using crystals with crystalline plane spacing $d_{0}$, a ring with a radius $r_{1}>r_{0}$, according to the Bragg law, will concentrate the same photon energies only if the crystalline plane spacing $\mathrm{d}_{1}$ is smaller than $\mathrm{d}_{0}$ or if a higher order is used. From (3), for materials with a cubic structure for which $d_{h k l}$ is inversely proportional to $\sqrt{h^{2}+k^{2}+l^{2}}$, the ring radii are proportional to this quantity.

As the diffraction efficiency decreases with increasing diffraction order $n$, a crystal in an exterior ring will add less efficient area to the lens than a crystal on an inner ring. However, since the number of crystals increases with the ring-radius, all rings will usually contribute about the same amount of efficient area to the lens. Using larger and larger Bragg angles with increasing ring radius allows the instrument to be relatively compact, featuring a shorter focal length than required if the above criterion was not adopted. An example of a narrow passband Laue lens, the balloon telescope CLAIRE, will be discussed below.

4.5.2. Broad Passband Laue Lenses. These lenses use the best combination of crystalline planes to cover in the most efficent way the lens passband. Lowest order planes, for example, (111), are preferred because we can exploit, in addition to their optimum diffraction efficiency, also the higher order diffraction of the same planes. The principle used for covering a broad energy band is the following. In the simple assumption that a single crystal material and crystal plane $(h k l)$ are used, assuming a ring-like geometry of the lens, different concentric rings focus slightly different energies because of the varying Bragg angle, and thus with several rings a broad energy band can be covered with the first-order diffraction (lens nominal energy passband). But, in addition to the first-order diffraction, higher order diffraction can be exploited that increases the effective area at higher energies, while also extending the passband. An example of the distribution of the various crystals and lattice planes in different rings for a broad passband lens (100$600 \mathrm{keV}$ ) made of mosaic crystals with $20 \mathrm{~m}$ focal length, is studied in [28] and it is shown in Figure 12.

Diffraction lenses with broad energy passbands were also developed and tested for low-energy X-rays since the sixties (e.g., Lindquist and Webber [36]). Today, photons up to $80 \mathrm{keV}$ can be efficiently focused thanks to the development of grazing incidence based on multilayer mirrors (see other papers in this issue). Above this upper threshold, the development of Laue lens telescopes becomes crucial for photon focusing.

\section{Optical Properties of a Lens}

The optical properties of a lens, for both on-axis and off-axis incident photons, have been investigated by means of Monte Carlo (MC) simulations $[11,31]$. For flat crystal tiles, the Point Spread Function (PSF) depends on the crystal size, on their mosaicity and on the accuracy of their positioning in the lens.

In Figure 13, we show the calculated on-axis Point Spread Functions for two cases: a ring-shaped lens of $40 \mathrm{~m}$ focal length and a spiral-shaped lens of $6 \mathrm{~m}$ focal length. In the first case, the lens has a $150-600 \mathrm{keV}$ energy passband and a crystal tile cross section of $10 \times 10 \mathrm{~mm}^{2}$, while in the second case it has a 70-300 keV passband and a crystal tile cross section of $15 \times 15 \mathrm{~mm}^{2}$. In both cases it is supposed that the crystals made of $\mathrm{Cu}(111)$, have a mosaic structure with 1 arcmin spread and they are perfectly oriented in the lens. The $6 \mathrm{~m}$ focal length lens has been proposed for a balloon experiment [33].

In the case of curved crystals, whose development is giving very satisfactory results (see Section 3.3), the expected PSF becomes very sharp, with a great advantage in terms of angular resolution and sensitivity. Assuming a lens of $15 \mathrm{~m}$ focal length made of crystals with a mosaic spread of 30 arcsec, the PSF obtained in the case of $15 \times 15 \mathrm{~mm}^{2}$ flat mosaic crystals and that obtained in the case of curved crystals with a curvature radius of $30 \mathrm{~m}$ (2 times the focal length) are shown in Figure 15. The difference between the two PSFs is outstanding. In the case of curved crystals we expect an angular resolution of 20 arcsec and a sensitivity higher than the corresponding lens with flat crystals by a factor of about 10.

In Figure 14, for flat crystal tiles of $15 \times 15 \mathrm{~mm}^{2}$, we show the expected PSF of the $40 \mathrm{~m}$ focal length lens when three sources are in the Field of View (FOV), with one of the sources on-axis and the other two off-axis. As can 


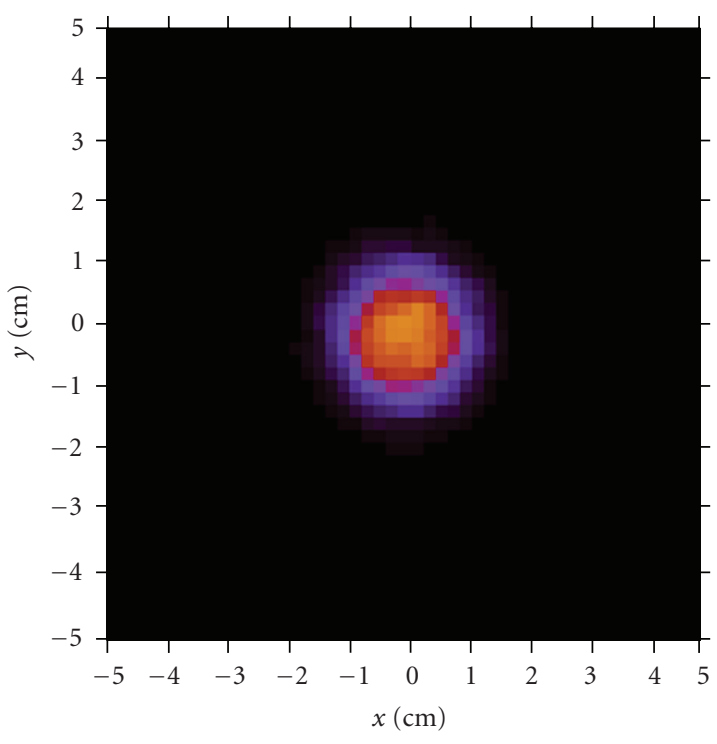

(a)

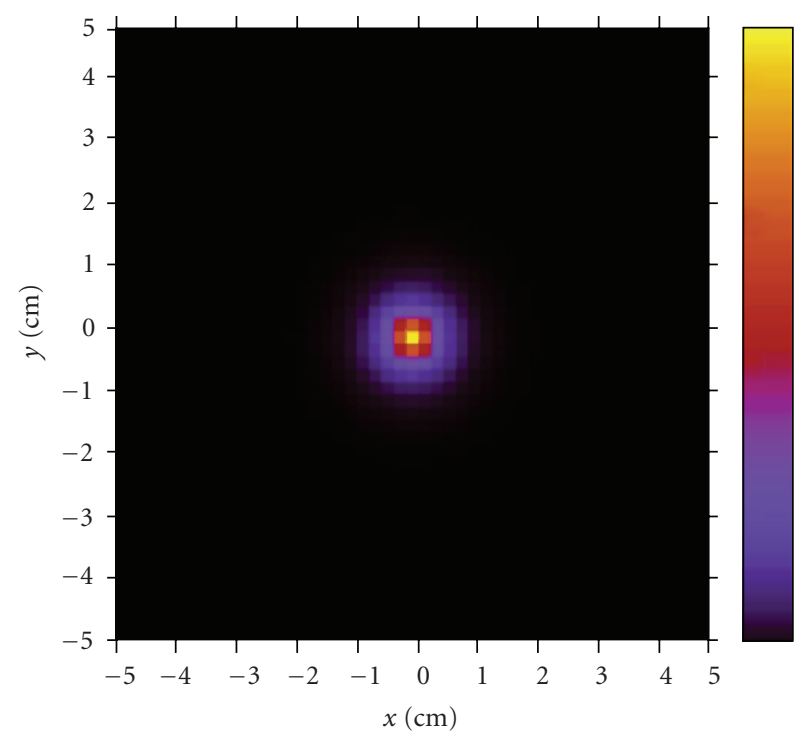

(b)

FIGURE 15: On-axis response function (PSF) of a lens of $15 \mathrm{~m}$ focal length made of mosaic crystals with 30 arcsec spread, for an on-axis source. (a) Flat crystals with cross section of $15 \times 15 \mathrm{~mm}^{2}$. (b) Curved crystals with a curvature radius of $30 \mathrm{~m}$.

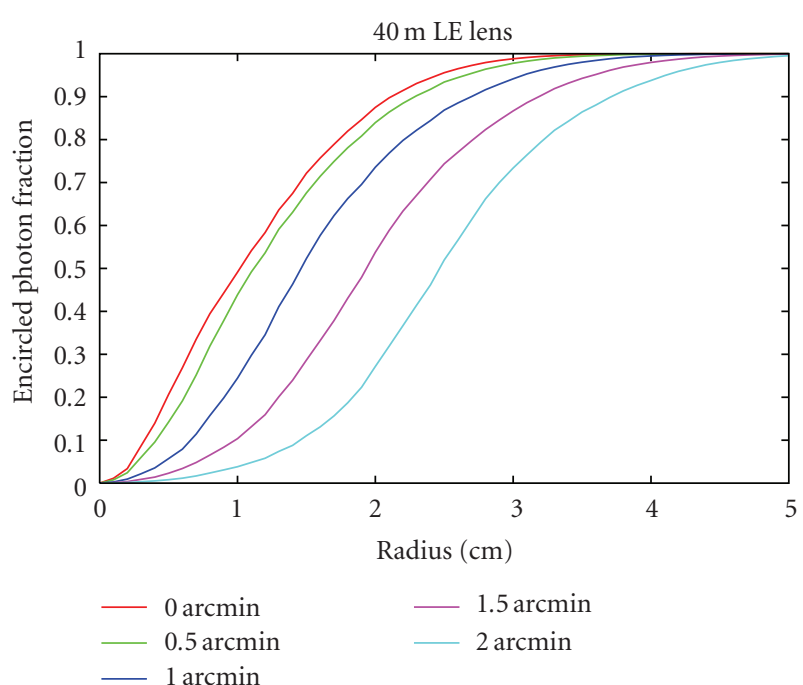

FIGURE 16: Cumulative distribution of photons with the distance from the lens focus, for different values of the mosaic spread, $40 \mathrm{~m}$ focal length lens, at about $100 \mathrm{keV}$.

be seen, in the case of off-axis sources the image has a ring shape centered in the on-axis source image, with inner radius that increases with the offset angle, and a nonuniform distribution of the reflected photons with azimuth. It is found that the integrated number of photons focused by the lens does not significantly vary from an on-axis source to an off-axis source, but they are spread over an increasing area. As a consequence, in principle the FOV of the lens is determined by the detector radius, but, taking into account that the focused photons from sources at increasing offset spread over an increasing area, the lens sensitivity decreases

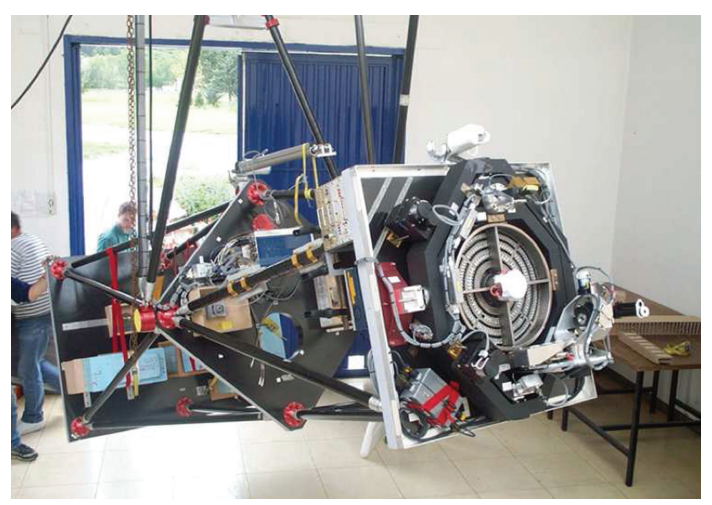

Figure 17: The CLAIRE telescope at the Gap-Tallard balloon base during the 2001 balloon campaign. On the first platform, the gamma-ray lens in its two-axes gimbal.

with the source offset. The azimuthal nonuniformity of the PSF for off-axis sources can be usefully exploited, because it gives information on the azimuthal source direction. The angular resolution, in addition to the size of the flat crystal tiles, depends on the mosaic spread and on the misalignments of the lens crystal tiles. For the lens image shown in Figure 14, the angular resolution is of the order of, or even better than, 1 arcmin.

Figure 16 shows the cumulative distribution of the onaxis photons with the distance from the lens focus, for different values of the mosaic spread, in the case of a $40 \mathrm{~m}$ focal length lens made of mosaic crystals of $\mathrm{Cu}(111)$ with 15 $\times 15 \mathrm{~mm}^{2}$ flat crystal tile cross section. As can be seen, for a low mosaic spread, the distribution is driven by the crystal size, while for large spreads, it is mainly driven by this spread. 


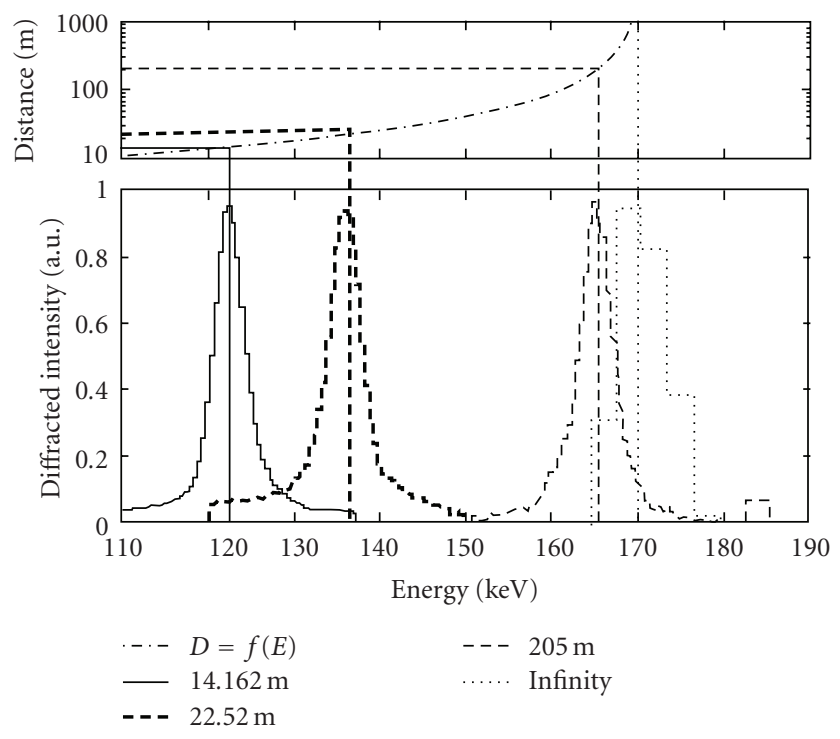

FIGURE 18: Recorded spectra for various source distances (lower graph). The upper graph represents the distance of the source as a function of diffracted energy. The vertical lines show the theoretical corresponding energies. $14.162 \mathrm{~m}$ corresponds to the tuning distance $\left(\mathrm{E}_{\mathrm{th}}=122.29 \mathrm{keV}\right)$. The measurement at $22.52 \mathrm{~m}$ $\left(\mathrm{E}_{\mathrm{th}}=136.5 \mathrm{keV}\right)$ was performed on the optical bench at CESR with a partially tuned lens. $205 \mathrm{~m}$ is the distance of the generator on the long distance test range $\left(\mathrm{E}_{\mathrm{th}}=165.5 \mathrm{keV}\right)$. The peak for an infinite distance is taken from the stratospheric flight of June 2001.

\section{The Development of Laue Lenses}

Two key issues have to be faced in order build a Laue lens:

(i) Development of technologies for the mass production of suitable crystals (mosaic and/or curved crystals) in a reasonable time, consistent with that of preparation of a space mission.

(ii) Development of a technology for assembling, in an equally reasonable time, thousands of crystal tiles in a lens with the proper orientation accuracy, that largely depends on the focal length.

Laue lens developments are being carried out in different institutions. We summarize here the major results obtained in our institutes.

6.1. CLAIRE-a Narrow Passband Laue Lens Experiment. The objective of the R \& D (Research and Development) project CLAIRE (French word that means "clear") [37] was to demonstrate that a prototype Laue lens can work under space conditions, measuring its performance by observing an astrophysical target. The CLAIRE telescope was flown twice $(2000,2001)$ on a stratospheric balloon by the French Space Agency CNES. CLAIRE's Laue lens was further tested on a $205 \mathrm{~m}$ long optical bench in 2003 [38]. The project involved research groups from CESR (Centre d'Etude Spatiale des Rayonnements) Toulouse, University of Birmingham, Institute Laue-Langevin (ILL) Grenoble, IEEC (Institut d'Estudis Espacials de Catalunya) Barcelona, and

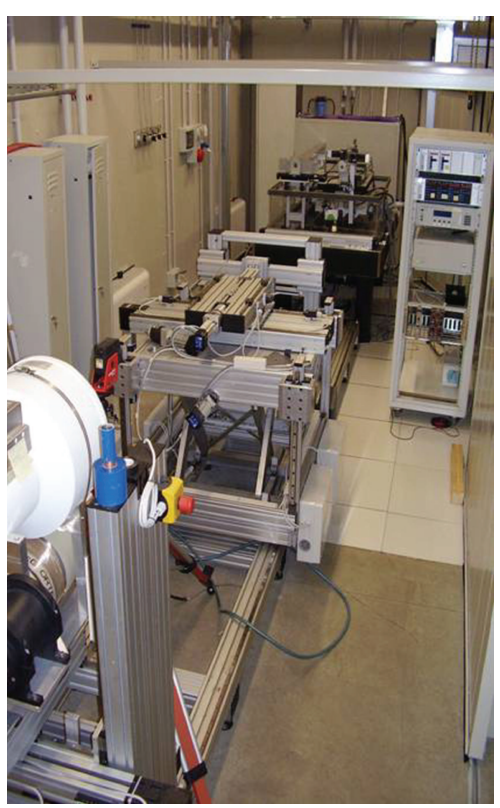

Figure 19: A view of the current configuration of the apparatus for the lens assembling. The apparatus is located in the LARIX lab of the University of Ferrara.

Argonne National Laboratory Chicago. CLAIRE's narrow passband lens consisted of 556 crystals (see Table 1) mounted on eight rings of a $45 \mathrm{~cm}$ diameter Titanium frame. In each ring $i$, the combination of the crystal plane spacing $d_{i}$ and the Bragg angle $\theta_{\mathrm{Bi}}$ results in the concentration of $170 \mathrm{keV}$ photons onto a common focal spot of $1.5 \mathrm{~cm}$ diameter at $279 \mathrm{~cm}$ behind the lens.

CLAIRE's crystals were produced by $N$. Abrosimov at the Institut für Kristallzüchtung (IKZ) in Berlin. The Germanium-rich $\mathrm{Ge}_{1-x} \mathrm{Si}_{x}$ crystals $(x \approx 0.02)$ were grown by a modified Czochralski technique using Silicon feeding rods to replenish the loss of $\mathrm{Si}$ in the melt during the growth. The mosaicities of the $\mathrm{Ge}_{1-x} \mathrm{Si}_{x}$ crystals range between roughly 30 arcsec and 2 arcmin, leading to a field of view of about $1.5 \mathrm{arcmin}$ and a diffracted energy bandwidth of about $3 \mathrm{keV}$ at $170 \mathrm{keV}$. A correlative study between crystal structure, mosaicity and diffraction efficiency of the CLAIRE crystals is presented in Abrosimov et al. [39]. After cutting the crystal ingots at IKZ Berlin, most of the crystal tiles were characterized (mosaicity) at the Hard X-Ray Diffractometer of ILL Grenoble.

At CESR Toulouse, the individual crystal tiles were mounted on flexible aluminium supports, which in turn are mounted on the lens frame. The reinforced $45 \mathrm{~cm}$ diameter titanium frame that holds up to 576 crystals on 8 rings was designed and manufactured at the Argonne National Laboratory, Argonne, USA. The tuning of the lens consisted of tilting each crystal tile to the appropriate Bragg angle so that the diffracting energy was $170 \mathrm{keV}$ for a source at infinity. Instead of directly calibrating the lens for a parallel beam of $170 \mathrm{keV}$ photons, crystal tuning on the $20 \mathrm{~m}$ optical bench at CESR used a $150 \mathrm{kV}$ X-ray generator situated on the lens optical axis at a distance of $14.16 \mathrm{~m}$. At this 


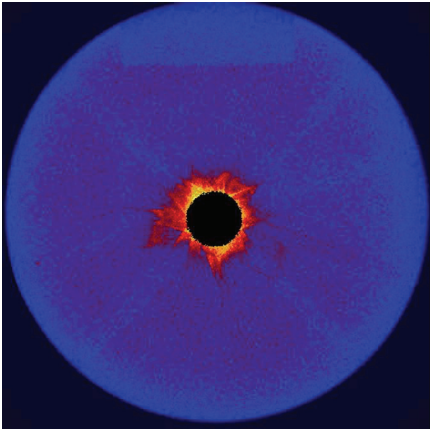

(a)

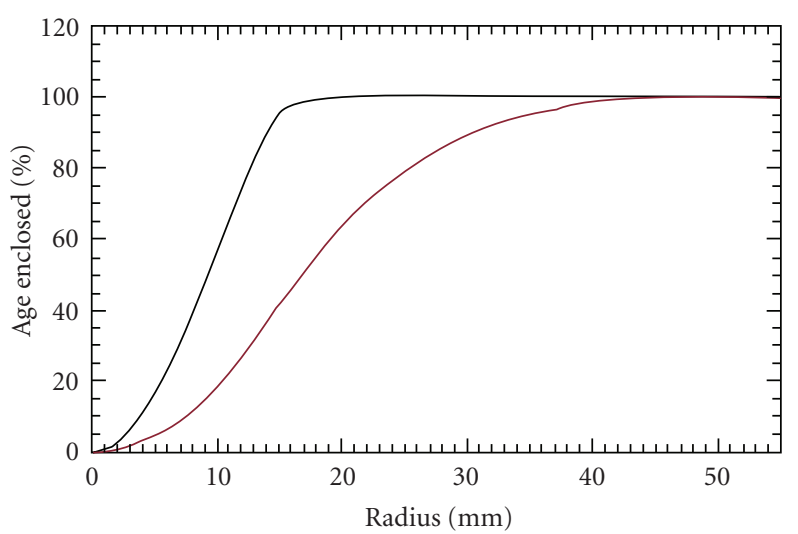

(b)

FIgURe 20: (a) Difference between the PSF measured and that obtained with a Monte Carlo code by assuming a perfect positioning of the crystal tiles in the lens. (b) Cumulative distribution of the focused photons as a function of radial distance from the focal point. Black line: expected distribution in the case of a perfect positioning of the crystal tiles in the lens. Red line: measured distribution. Reprinted from [34].

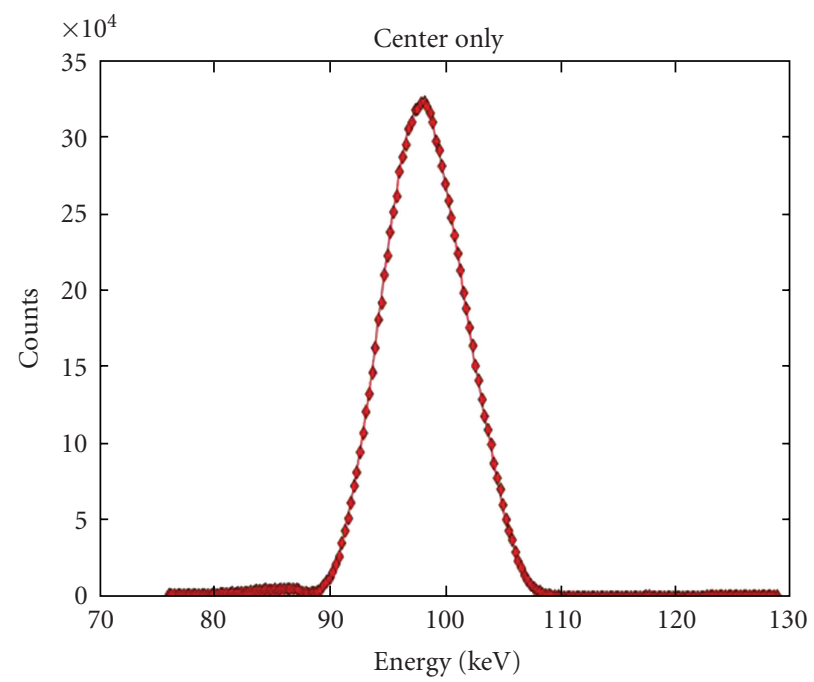

(a)

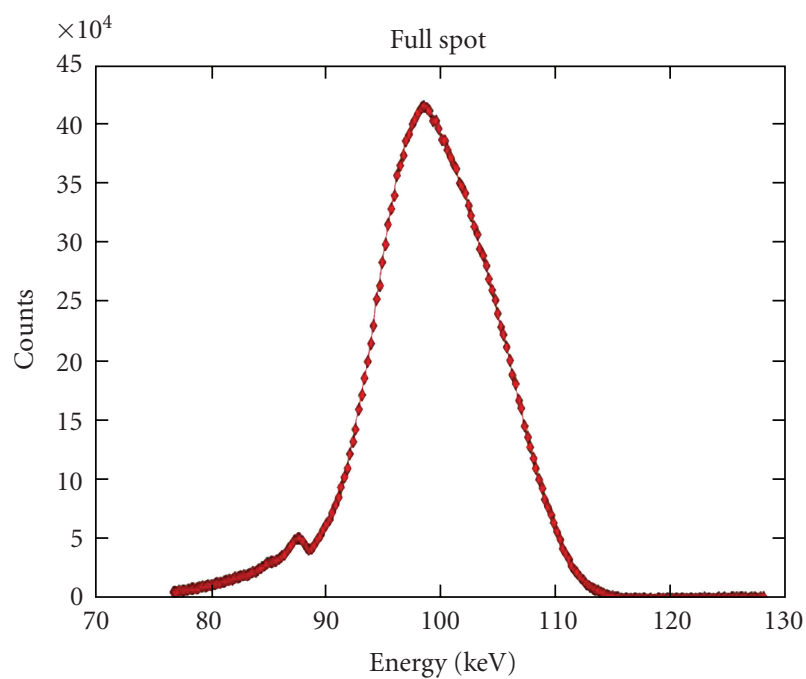

(b)

Figure 21: (a) Photon spectrum of the region (see left panel of Figure 20) in which all the reflected photons are expected to be found in the case of a perfect mounting of the crystal tiles in the lens. (b) Spectrum of all reflected photons. Reprinted from [34].

distance, a crystal was correctly tuned ( $170 \mathrm{keV}$ at infinity) if it diffracted $122.28 \mathrm{keV}$ photons. A coaligned mask (brasslead sandwich) of the size of the entire lens, placed on the optical axis just in front of the lens, was used to select an individual crystal for tuning, while shielding already tuned crystals.

The resulting geometric area of the CLAIRE lens was $511 \mathrm{~cm}^{2}$, the FOV and the passband were $90^{\prime \prime}$ and $\sim 3 \mathrm{keV}$, respectively. The photons were focused onto a small $3 \times$ 3 array of high-purity Germanium detectors, housed in a single cylindrical aluminum cryostat. Each of the single Ge bars was an $n$-type coaxial detector with dimensions of $1.5 \mathrm{~cm} \times 1.5 \mathrm{~cm} \times 4 \mathrm{~cm}$. Focusing onto such a small detector volume already results in very low background noise. The CLAIRE stabilization and pointing system was developed by the balloon division of the French space agency CNES.
CLAIRE's First Light. On June 14 2001, CLAIRE was launched by the balloon division of French Space Agency CNES from its base at Gap-Tallard in the French Alps (see Figure 17). The astrophysical target was the Crab Nebula. (While nuclear lines are the perfect astrophysical targets for narrow passband Laue lenses, the balloon test flight ironically required the observation of a continuum spectrum.) With a mere 72 minutes of the flight having satisfactory pointing, CLAIRE nevertheless collected 33 photons from the Crab Nebula. This $3 \sigma$ detection has been validated by ground tests conducted at distances of $14.16 \mathrm{~m}, 22.52 \mathrm{~m}$ (CESR optical bench), and $205 \mathrm{~m}$ (long distance test, Ordis, Catalunia). Figure 18 shows the recorded spectra for these experiments. The energies of the centroids are in very good agreement with theory, slight departures from theoretical values (less than $0.5 \mathrm{keV}$ ) being the consequence of the incident spectrum 


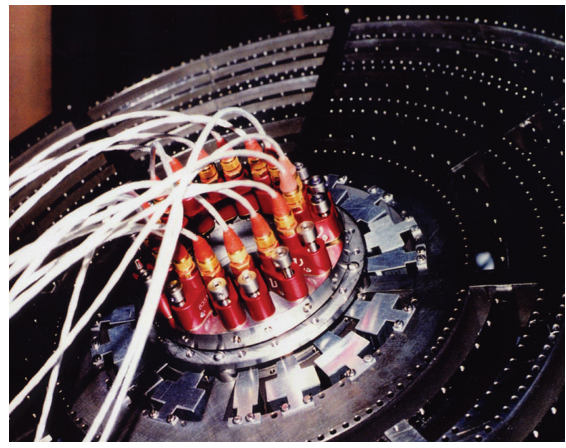

(a)

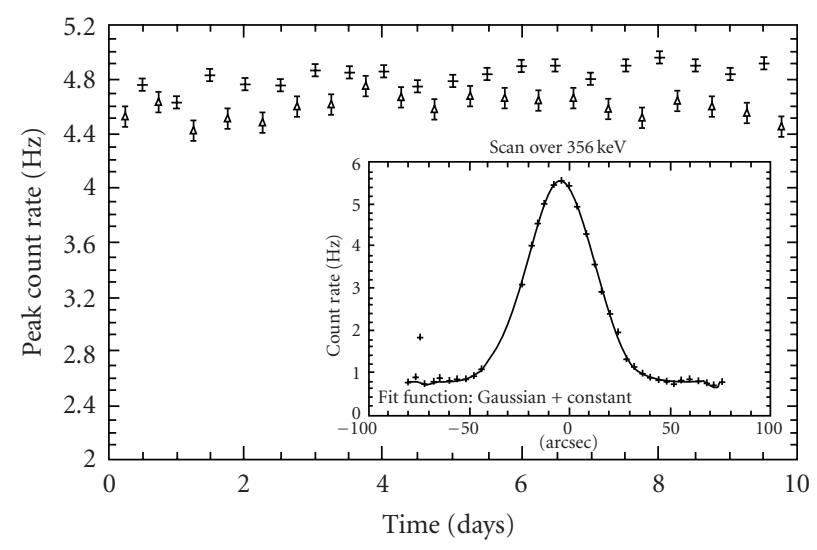

(b)

Figure 22: (a) Prototype tunable lens. (b) The evolution in time of the peak count rate when alternatively focusing $303 \mathrm{keV}$ (circles) and $356 \mathrm{keV}$ (crosses) $\gamma$-rays demonstrates the stability and reproducibility of the lens tuning.

TABLE 1: The crystalline plane, the crystal size, the number of crystals per ring as well as the ring radius, and the Bragg angle at $170 \mathrm{keV}$ are listed for each ring of the prototype crystal lens.

\begin{tabular}{|c|c|c|c|c|c|c|}
\hline ring & Reflection $[h k l]$ & $\mathrm{d}[h k l][\mathrm{A}]$ & Size $[\mathrm{mm}]$ & $\begin{array}{c}\text { Number of } \\
\text { crystals }\end{array}$ & Radius $[\mathrm{cm}]$ & $\begin{array}{c}\text { Bragg angle at } \\
170 \mathrm{keV}\end{array}$ \\
\hline 0 & 111 & 3.27 & $10 \times 10$ & 28 & 6.17 & 0.64 \\
\hline 1 & 220 & 2.00 & $10 \times 10$ & 52 & 10.08 & 1.04 \\
\hline 2 & 311 & 1.71 & $10 \times 10$ & 56 & 11.82 & 1.22 \\
\hline 3 & 400 & 1.41 & $10 \times 10$ & 72 & 14.26 & 1.48 \\
\hline 4 & 331 & 1.30 & $10 \times 7$ & 80 & 15.62 & 1.61 \\
\hline 5 & 422 & 1.15 & $10 \times 10$ & 96 & 17.47 & 1.81 \\
\hline 6 & 333 & 1.09 & $10 \times 7$ & 96 & 18.82 & 1.92 \\
\hline 7 & 440 & 1 & $10 \times 10$ & 104 & 20.17 & 2.09 \\
\hline
\end{tabular}

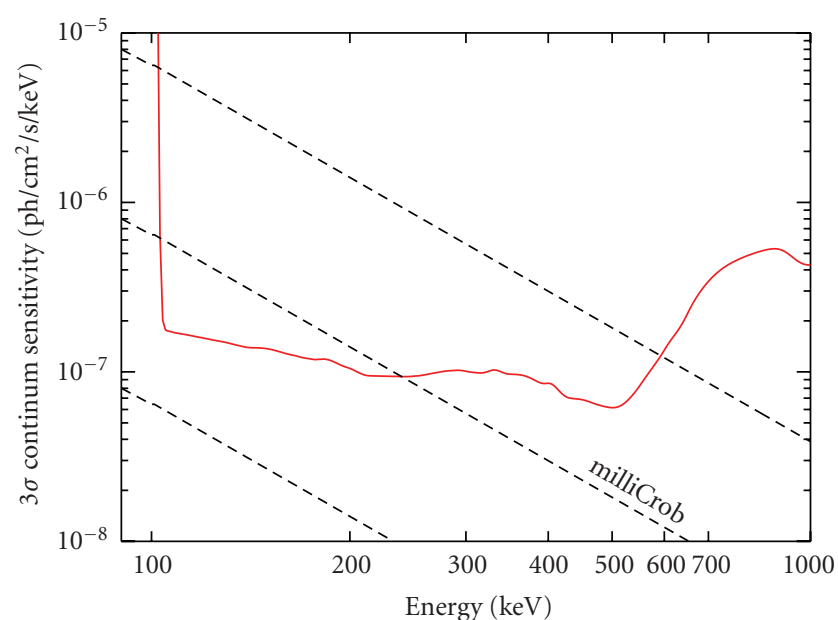

Figure 23: $3 \sigma$ continuum sensitivity $(\Delta E=E / 2)$ of the lens discussed in Section 4.5.2 for 100 ks observation time. Reprinted from [28].

shape, and/or the detector calibration drifts. The measured peak efficiencies of the ground experiments are in fairly good agreement with the Crab observation; when their efficiencies are rescaled for a polychromatic source at infinity, a peak efficiency of $9 \pm 1 \%$ is obtained. At first glance, this figure may seem rather modest; however, when considering the constraints on a compact balloon instrument, the result is actually very positive. The short focal length leads to a lens with the outermost rings occupied by crystals with high reflection orders $n$ (see (1)); the outermost rings ( (333) and (440) crystals) are roughly 4 times less efficient than the innermost rings ( (111) and (220) crystals). Note that the largest numbers of crystals are situated in the outer rings where efficiencies are unfortunately lowest. A future space instrument will allow longer focal lengths and hence only low-order crystalline planes with the highest efficiencies would be used. Also, the quality of the CLAIRE crystals was quite heterogeneous the efficiency of the individual crystals within a ring varied by factors of 2 to 10, depending on the ring! However, the CLAIRE lens contained crystals as efficient as the Darwin model predicts: the best crystals of the lens showed peak efficiencies well above $20 \%$.

CLAIRE's balloon flight provided the first observation of an astrophysical source with a gamma-ray lens. In combination with the long-distance test on the ground, these results validate the theoretical models and demonstrate the principle of Laue lens. Moreover, CLAIRE's stratospheric 


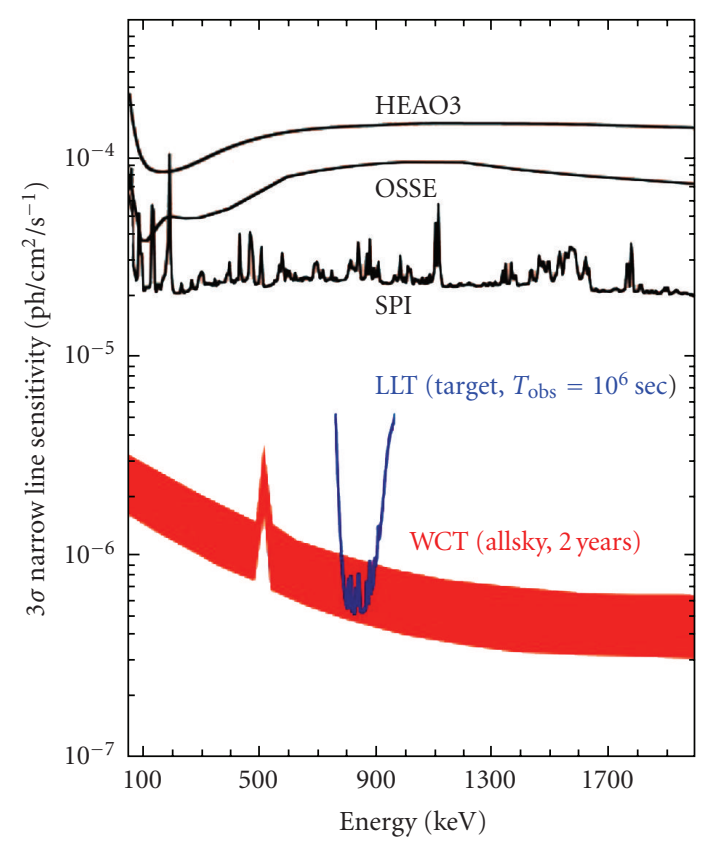

FIgUre 24: Sensitivity estimation of DUAL's Laue Lens Telescope (LLT, blue curve), enabling study of SNe Ia out to $50-80 \mathrm{Mpc}$, for dozens of potential targets each year. Also shown is the estimated survey sensitivity (two years) of the Wide Field Compton Telescope (WCT, red curve) which serves as a focal plane detector of the LLT.

flight represents a first demonstration of the Laue lens technology in a space environment.

6.2. Current Crystal Development. The crystal development status is discussed in detail in [24, 40]. Mosaic crystals of Copper are developed and produced at ILL [32]; gradient composition crystals of $\mathrm{Si}_{1-x} \mathrm{Ge}_{x}$ alloy with $x$ increasing along the crystal growth axis (curved crystal) are produced at IKZ [39]. Crystal developments for Laue lenses are being undertaken in Italy: mosaic crystals at the CNR-IMEM Institute and curved crystals at the Physics Department, University of Ferrara (see Figure 6).

6.3. Current Lens Assembly Technology Development. Currently new lens assembly technologies are being developed at the University of Ferrara, for building broad passband (70/100-600 keV) Laue lenses, and at CESR for building high-energy narrow passband (800-900 keV) Laue lenses.

The crystal tile positioning accuracy in the lens is the most critical issue. It depends on the mosaic spread and the focal length. Longer focal lengths require better positioning accuracies, thus the development is more challenging for lenses working at the highest energies.

\subsubsection{Assembly Technology Status for Broad Passband Laue} Lenses. A technology for assembling crystal tiles for a moderately short ( $\leq 10-15 \mathrm{~m}$ ) focal length lens is at an advanced stage of development at the University of Ferrara [34, 41, 42]. It does not require any mechanism for a fine adjustment of the crystal orientation once the crystal is positioned in the lens frame. Using this technology, a first lens prototype with $6 \mathrm{~m}$ focal length has already been developed and tested. It makes use of mosaic crystals of $\mathrm{Cu}(111)$.

The technique adopted is described in detail in [34, 41]. It makes use of a countermask provided with holes, two for each crystal tile. Each tile is positioned on the countermask by means of two cylindrical pins, rigidly glued to the crystal tile, that are inserted in the countermask holes. The pin direction and the axis of the average lattice plane of each crystal tile have to be exactly orthogonal. The hole axis direction constrains the energy of the photons diffracted by the tile, while the relative position of the two holes in the countermask establishes the azimuthal orientation of the axis of the crystal lattice plane. This axis has to cross the lens axis.

Depending on the direction of the hole axes in the countermask, the desired geometry of lens can be obtained. In the case of a lens for space astronomy, the hole axes have to be directed toward the center of curvature of the lens. In the case of the developed prototype, the hole axes were set parallel to the lens axis for the quick test of the lens with an $\mathrm{X}$-ray tube, that provides a divergent X-ray beam.

Once all the crystal tiles are placed on the counter-mask, a frame is glued to the entire set of the crystals. Then the lens frame, along with the crystals, is separated from the counter-mask and from the pins. In the case of the first prototype, instead of using a chemical attack in order to separate the countermask from the lens as foreseen in the project, a mechanical separation was attempted.

The first developed prototype is made of a $36 \mathrm{~cm}$ diameter ring of 20 mosaic crystal tiles. The mosaic spread of the used crystals ranges from $\sim 2.5$ to $\sim 3.5 \mathrm{arcmin}$. The tile cross-section is $15 \times 15 \mathrm{~mm}^{2}$ while its thickness is $2 \mathrm{~mm}$. The lens frame is made of carbon fiber composite with a total thickness of $1 \mathrm{~mm}$.

The X-ray beam used, first to assemble the crystals and then to test the complete lens, is that available in the LARIX (LArge Italian hard X-ray) facility of the University of Ferrara. For an LARIX description see [43]. A view of the experimental apparatus in the current configuration is shown in Figure 19.

The prototype was thoroughly tested using the polychromatic X-ray beam described above, as reported in [34]. Figure 20 shows the difference between the measured PSF and that obtained from a simulation, in which a perfect positioning of the crystals in the lens was assumed. As can be seen, only the center part of the measured image (i.e., the black region) is subtracted by the simulated image. The corona still visible in the difference image is the result of the cumulative error (mainly that due to the mechanical separation of the lens from the countermask) during the lens assembly process.

The disagreement between the measured and the expected PSF is also apparent by comparing the cumulative distribution of the photons with the distance from the lens focus (see Figure 20(b)). As can be seen, the PSF radius at which the expected fraction of focused photons reach the saturation $(16 \mathrm{~mm})$ corresponds to $\sim 60 \%$ of the measured fraction. 
The spectrum of the photons focused by the developed prototype is shown in Figure 21, where we compare the measured spectrum of the central region (i.e., photons in the black region of the left panel of Figure 20) with the spectrum of all reflected photons. As can also be seen from this figure, the centroid of the spectrum of the central region achieves an intensity level 0.8 times that of the peak spectrum of all reflected photons.

A new prototype is being developed that takes into account the experience gained constructing the first one [42].

6.4. $R$ \& $D$ for a Tunable Narrow Passband Lens. Observing in only one narrow energy band might be considered too much of a handicap for a space instrument. In the framework of an R \& D project for the French Space Agency CNES, a tunable $\gamma$-ray lens prototype (Figure 22(a)) was developed and demonstrated [44]. The capability to observe more than one astrophysical line with a narrow passband Laue lens requires the tuning of two parameters: the Bragg angle $\theta_{\mathrm{B}}$ and the focal length $f$. While the length $f$ will have to be controlled to within $\sim 1 \mathrm{~cm}$, the precision of the crystal inclination has to be better than the mosaic structure of the crystals. In the setup of [44], each crystal was tuned by using piezo-driven actuators to change the crystal inclination, and an eddy-current sensor to determine the current position (Figure 22(a)). The resolution of the control-loop permitted an angular resolution of $0.1-0.4$ arcsec. The stability was found to be better than 0.8 arcsec per day and the reproducibility of a particular tuning better than 5 arcsec over a 10-day period (Figure 22(b)).

\section{Prospects and Conclusions}

A big effort has already been invested in the development of focusing Laue lenses for gamma-ray astronomy $(>70 / 100 \mathrm{keV})$. Thanks to the most recent developments, Laue lenses with short focal length $(10-15 \mathrm{~m})$ are already feasible. The major task now in progress is the development of the crystals needed to optimize the lens effective area. A project "LAUE," supported by the Italian Space Agency, has just started in Italy (main contractor DTM, Modena) for the development of both suitable Laue lens crystals and an advanced assembly technology for long focal length lenses (up to $100 \mathrm{~m}$ ).

Thus far, the major limit to the launch of a Laue lens gamma-ray telescope has been the need of long focal lengths $(20-100 \mathrm{~m})$, that implies the use of two satellites in formation flying, one for the lens and the other for the focal plane detector. The development of extendable booms up to $20 \mathrm{~m}$, the optimization of the lens effective area and the limitation of the lens passband to lower energies, all make realistic the prospect of broad band satellite missions that could join together multilayer mirrors and Laue lenses to extend the focusing band up to several hundreds of $\mathrm{keV}$.

As an example, the $10^{5} \mathrm{~s}$ continuum sensitivity of a Laue lens made of mosaic crystals, that was investigated in
[28] and mentioned above (see Section 4.5.2), is shown in Figure 23. The use of curved crystals can further increase the lens sensitivity.

In the framework of formation flying missions, the mission concept DUAL [45] is currently under study by a consortium of institutes from Europe, Japan and the USA. The DUAL mission is composed of a Wide-field Compton telescope (WCT) which carries out all-sky surveys, and a narrow band (800-900 keV) Laue-Lens Telescope (LLT) that simultaneously performs very deep observations of selected narrow-field targets. Combining a small Compton telescope with a Laue lens will permit the resolution of the apparently contradictory needs for a future gamma-ray mission: to map out large-scale distributions, monitor extreme accelerators, and measure the polarization of gamma-ray bursts with a small medium-sensitivity Compton Camera and simultaneously accomplish the stringent performance requirements for the specific science goal of SNla thanks to the Laue Lens. A compact, wide field (2-3 $\pi$ steradian) Compton telescope with a modest geometric area $\left(400-1600 \mathrm{~cm}^{2}\right)$ resulting in a effective area of $40-160 \mathrm{~cm}^{2}$ can fulfill the needs for the "all-sky science" and simultaneously serve as a focal plane detector for the LLT. A Laue lens that, in $10^{6} \mathrm{~s}$, can achieve sensitivities of $10^{-6}$ photons $\mathrm{cm}^{-2} \mathrm{~s}^{-1}$ for a $3 \%$ broadened line at $847 \mathrm{keV}$ can be made from $\mathrm{Au}, \mathrm{Ag}$, and $\mathrm{Cu}$ crystals, similar to those presently available. The model lens used for the sensitivity shown in Figure 24 would have a focal length of the order of $68 \mathrm{~m}$, a radius of $40-58 \mathrm{~cm}$, and a total mass of $40 \mathrm{~kg}$.

We expect that future broad band X-/gamma-ray missions for the deep study of nonthermal astrophysical processes above $100 \mathrm{keV}$, antimatter annihilation signatures, and nuclear lines from SN explosions will include Laue lenses.

\section{Acknowledgments}

F. Frontera acknowledges the financial support by the Italian Space Agency ASI. P. v. Ballmoos acknowledges continuing support from the French Space Agency CNES and is particularly grateful to the Balloon Division of CNES which built the pointing system and operated the CLAIRE flights from the launch to the gondola recovery. The authors also would like to thank the anonymous referees who have given valuable contributions to improve this paper.

\section{References}

[1] C. Winkler, N. Gehrels, V. Schönfelder et al., "First results from the INTEGRAL galactic plane scans," Astronomy \& Astrophysics, vol. 411, no. 1, pp. L349-L355, 2003.

[2] N. Gehrels, G. Chincarini, P. Giommi et al., "The Swift gamma-ray burst mission," Astrophysical Journal, vol. 611, no. 2 I, pp. 1005-1020, 2004.

[3] A. J. Bird, A. Bazzano, L. Bassani et al., "The fourth IBIS/ISGRI soft gamma-ray survey catalog," Astrophysical Journal Supplement Series, vol. 186, no. 1, pp. 1-9, 2010.

[4] G. Cusumano, V. La Parola, A. Segreto et al., "The Palermo Swift-BAT hard X-ray catalogue. II-results after 39 months 
of sky survey," Astronomy \& Astrophysics, vol. 510, article A48, 2010.

[5] G. Weidenspointner, G. Skinner, P. Jean et al., "An asymmetric distribution of positrons in the Galactic disk revealed by $\gamma$ rays," Nature, vol. 451, no. 7175, pp. 159-162, 2008.

[6] R. Diehl, H. Halloin, K. Kretschmer et al., "Radioactive ${ }^{26} \mathrm{Al}$ from massive stars in the Galaxy," Nature, vol. 439, no. 7072, pp. 45-47, 2006.

[7] F. Frontera, E. Costa, D. dal Fiume et al., "PDS experiment on board the BeppoSAX satellite: design and inflight performance results," in EUV, X-ray, and Gamma-Ray Instrumentation for Astronomy VIII, O. H. Siegmund and M. A. Gummin, Eds., vol. 3114 of Proceedings of SPIE, pp. 206215, San Diego, Calif, USA, July 1997.

[8] P. Ubertini, F. Lebrun, G. Di Cocco et al., "IBIS: the Imager onboard INTEGRAL," Astronomy \& Astrophysics, vol. 411, no. 1, pp. L131-L139, 2003.

[9] J. Knödlseder, P. von Ballmoos, F. Frontera et al., "GRI: focusing on the evolving violent Universe," in Optics for EUV, X-ray, and Gamma-Ray Astronomy III, S. L. O'Dell and G. Pareschi, Eds., vol. 6688 of Proceedings of SPIE, San Diego, Calif, USA, August 2007.

[10] F. Frontera, A. Pisa, P. de Chiara et al., "Exploring the hard $\mathrm{X}$-/soft gamma-ray continuum spectra with Laue lenses," in Proceedings of the 39th ESLAB Symposium, F. Favata, J. SanzForcada, A. Giménez, and B. Battrick, Eds., no. 588, pp. 323 326, Noordwijk, The Netherlands, April 2005.

[11] F. Frontera, A. Pisa, V. Carassiti et al., "Gamma-ray lens development status for a European gamma-ray imager," in Space Telescopes and Instrumentation II: Ultraviolet to Gamma Ray, M. J. L. Turner and G. Hasinger, Eds., vol. 6266 of Proceedings of SPIE, May 2006.

[12] J. Knödlseder, "GRI: the gamma-ray imager mission," in Space Telescopes and Instrumentation II: Ultraviolet to Gamma Ray, M. J. L. Turner and G. Hasinger, Eds., vol. 6266 of Proceedings of SPIE, Orlando, Fla, USA, May 2006.

[13] D. Götz, S. Mereghetti, A. Tiengo, and P. Esposito, "Magnetars as persistent hard X-ray sources: INTEGRAL discovery of a hard tail in SGR 1900+14," Astronomy \& Astrophysics, vol. 449, no. 2, pp. L31-L34, 2006.

[14] L. Kuiper, W. Hermsen, P. R. den Hartog, and W. Collmar, "Discovery of luminous pulsed hard X-ray emission from anomalous X-ray pulsars 1RXS J1708-4009, 4U 0142+61, and 1E $2259+586$ by INTEGRAL and RXTE," Astrophysical Journal, vol. 645, no. 1, pp. 556-575, 2006.

[15] G. C. Perola, G. Matt, M. Cappi et al., "Compton reflection and iron fluorescence in BeppoSAX observations of Seyfert type 1 galaxies," Astronomy \& Astrophysics, vol. 389, no. 3, pp. 802-811, 2002.

[16] G. Risaliti, "The BeppoSAX view of bright compton-thin Seyfert 2 galaxies," Astronomy \& Astrophysics, vol. 386, no. 2, pp. 379-398, 2002.

[17] G. Ghisellini, R. D. Ceca, M. Volonteri et al., "Chasing the heaviest black holes of jetted active galactic nuclei," Monthly Notices of the Royal Astronomical Society, vol. 405, no. 1, pp. 387-400, 2010.

[18] Y. Rephaeli, J. Nevalainen, T. Ohashi, and A. M. Bykov, "Nonthermal phenomena in clusters of galaxies," Space Science Reviews, vol. 134, no. 1-4, pp. 71-92, 2008.

[19] R. Gilli, A. Comastri, and G. Hasinger, "The synthesis of the cosmic X-ray background in the Chandra and XMM-Newton era," Astronomy \& Astrophysics, vol. 463, no. 1, pp. 79-96, 2007.
[20] A. Goldwurm, J. Ballet, B. Cordier et al., "Sigma/GRANAT soft gamma-ray observations of the X-ray nova in Muscadiscovery of positron annihilation emission line," Astrophysical Journal, vol. 389, pp. L79-L82, 1992.

[21] W. H. Zachariasen, Theory of X-rays Diffraction in Crystals, John Wiley \& Sons, New York, NY, USA, 1945.

[22] D. Pellicciotta, F. Frontera, G. Loffredo et al., "Laue lens development for hard X-rays (>60 keV)," IEEE Transactions on Nuclear Science, vol. 53, no. 1, pp. 253-258, 2006.

[23] A. Authier, Dynamical Theory of X-ray Diffraction, Oxford University Press, Oxford, UK, 2001.

[24] N. Barriére, J. Rousselle, P. von Ballmoos et al., "Experimental and theoretical study of the diffraction properties of various crystals for the realization of a soft gamma-ray Laue lens," Journal of Applied Crystallography, vol. 42, no. 5, pp. 834-845, 2009.

[25] S. Keitel, C. Malgrange, T. Niemöller, and J. R. Schneider, "Diffraction of 100 to $200 \mathrm{keV} \mathrm{X}$-rays from an $\mathrm{Si}_{1-x} \mathrm{Ge}_{x}$ gradient crystal: comparison with results from dynamical theory," Acta Crystallographica A, vol. 55, no. 5, pp. 855-863, 1999.

[26] C. Malgrange, "X-ray propagation in distorted crystals: from dynamical to kinematical theory," Crystal Research and Technology, vol. 37, no. 7, pp. 654-662, 2002.

[27] N. Barriere, Developpement d'une lentille de Laue pour l'astrophysique nucleaire, Ph.D. thesis, University of Toulouse, Toulouse, France, 2008.

[28] N. M. Barrière, L. Natalucci, N. Abrosimov et al., "Soft gamma-ray optics: new Laue lens design and performance estimates," in Optics for EUV, X-ray, and Gamma-Ray Astronomy IV, vol. 7437 of Proceedings of SPIE, San Diego, Calif, USA, August 2009.

[29] A. Pisa, F. Frontera, P. de Chiara et al., "Feasibility study of a Laue lens for hard X-rays for space astronomy," in Advances in Computational Methods for X-ray and Neutron Optics, M. Sanchez del Rio, Ed., vol. 5536 of Proceedings of SPIE, pp. 3948, Denver, Colo, USA, August 2004.

[30] A. Pisa, F. Frontera, P. de Chiara et al., "Development status of a Laue lens for high energy X-rays (>60 keV)," in Optics for EUV, X-ray, and Gamma-Ray Astronomy II, O. Citterio, S. L. O'Dell et al., Eds., vol. 5900 of Proceedings of SPIE, pp. 1-10, San Diego, Calif, USA, August 2005.

[31] A. Pisa, F. Frontera, G. Loffredo, D. Pellicciotta, and N. Auricchio, "Optical properties of Laue lenses for hard X-rays (>60 keV)," Experimental Astronomy, vol. 20, no. 1-3, pp. 219228, 2005.

[32] P. Courtois, K. H. Andersen, and P. Bastie, "Copper mosaic crystals for Laue lenses," Experimental Astronomy, vol. 20, no. 1-3, pp. 195-200, 2005.

[33] F. Frontera, G. Loffredo, A. Pisa et al., "A gamma-ray Laue lens focusing telescope aboard a balloon experiment," Memorie della Societa Astronomica Italiana, vol. 79, pp. 807-811, 2008.

[34] F. Frontera, G. Loffredo, A. Pisa et al., "Focusing of gammarays with Laue lenses: first results," in Space Telescopes and Instrumentation 2008: Ultraviolet to Gamma Ray, vol. 7011 of Proceedings of SPIE, Marseille, France, June 2008.

[35] N. Lund, "A study of focusing telescopes for soft gamma rays," Experimental Astronomy, vol. 2, no. 5, pp. 259-273, 1992.

[36] T. R. Lindquist and W. R. Webber, "A focusing X-ray telescope for use in the study of extraterrestrial X-ray sources in the energy range 20-140 keV," Canadian Journal of Physics, vol. 46, p. $1103,1968$. 
[37] P. von Ballmoos, H. Halloin, J. Evrard et al., "CLAIRE: first light for a gamma-ray lens,” Experimental Astronomy, vol. 20, no. 1-3, pp. 253-267, 2005.

[38] J. M. Alvarez, H. Halloin, M. Hernanz et al., "Long distance test of the CLAIRE gamma-ray lens," in Proceedings of the 5th INTEGRAL Workshop on The INTEGRAL Universe, pp. 757760, Munich, Germany, February 2004.

[39] N. V. Abrosimov, "Mosaic and gradient SiGe single crystals for gamma ray Laue lenses," Experimental Astronomy, vol. 20, no. 1-3, pp. 185-194, 2005.

[40] J. Rousselle, P. V. Ballmoos, N. Barrière et al., "High-Z crystals for gamma-ray optics," in Optics for EUV, X-ray, and GammaRay Astronomy IV, vol. 7437 of Proceedings of SPIE, San Diego, Calif, USA, August 2009.

[41] F. Frontera, G. Loffredo, A. Pisa et al., "Development status of a Laue lens project for gamma-ray astronomy," in Optics for EUV, X-ray, and Gamma-Ray Astronomy III, S. L. O'Dell, G. Pareschi et al., Eds., vol. 6688 of Proceedings of SPIE, San Diego, Calif, USA, August 2007.

[42] F. Ferrari, F. Frontera, G. Loffredo et al., "New results on focusing of gamma-rays with Laue lenses," in Optics for EUV, $X$-ray, and Gamma-Ray Astronomy IV, vol. 7437 of Proceedings of SPIE, San Diego, Calif, USA, August 2009.

[43] G. Loffredo, F. Frontera, D. Pellicciotta et al., "The Ferrara hard X-ray facility for testing/calibrating hard X-ray focusing telescopes," Experimental Astronomy, vol. 20, no. 1-3, pp. 413420, 2005.

[44] A. Kohnle, R. Smither, T. Graber, P. von Ballmoos, P. Laporte, and J. F. Olive, "Realization of a tunable crystal lens as an instrument to focus gamma rays," Nuclear Instruments and Methods in Physics Research A, vol. 408, no. 2-3, pp. 553-561, 1998.

[45] P. von Ballmoos, T. Takahashi, and S. E. Boggs, "A DUAL mission for nuclear astrophysics," Nuclear Instruments and Methods in Physics Research A, vol. 623, no. 1, pp. 431-433, 2010. 